\title{
Advanced computational and experimental techniques for nacelle liner performance evaluation
}

\author{
Carl H. Gerhold*, Michael G. Jones ${ }^{\dagger}$, Martha C. Brown ${ }^{\star}$, Douglas Nark ${ }^{\S}$ \\ NASA Langley Research Center, Hampton, Virginia
}

The Curved Duct Test Rig (CDTR) has been developed to investigate sound propagation through a duct of size comparable to the aft bypass duct of typical aircraft engines. The axial dimension of the bypass duct is often curved and this geometric characteristic is captured in the CDTR. The semiannular bypass duct is simulated by a rectangular test section in which the height corresponds to the circumferential dimension and the width corresponds to the radial dimension. The liner samples are perforate over honeycomb core and are installed on the side walls of the test section. The top and bottom surfaces of the test section are acoustically rigid to simulate a hard wall bifurcation or pylon. A unique feature of the CDTR is the control system that generates sound incident on the liner test section in specific modes. Uniform air flow, at ambient temperature and flow speed Mach 0.275, is introduced through the duct. Experiments to investigate configuration effects such as curvature along the flow path on the acoustic performance of a sample liner are performed in the CDTR and reported in this paper. Combinations of treated and acoustically rigid side walls are investigated. The scattering of modes of the incident wave, both by the curvature and by the asymmetry of wall treatment, is demonstrated in the experimental results. The effect that mode scattering has on total acoustic effectiveness of the liner treatment is also shown. Comparisons of measured liner attenuation with numerical results predicted by an analytic model based on the parabolic approximation to the convected Helmholtz equation are reported. The spectra of attenuation produced by the analytic model are similar to experimental results for both walls treated, straight and curved flow path, with plane wave and higher order modes incident. The numerical model is used to define the optimized resistance and reactance of a liner that significantly improves liner attenuation in the frequency range 1900-2400 $\mathrm{Hz}$. A liner impedance descriptor is used to determine the liner parameters that achieve the optimum impedance.

\section{Introduction}

Significant strides have been made in the reduction of aircraft engine noise in the past 50 years. One particularly notable design innovation is the high bypass ratio engine. This engine configuration uses the low pressure turbine to drive a large diameter fan, and it is the fan that provides most of the engine thrust. While the turbine exhaust noise dominates on take-off, the fan is the major noise source on approach with a noise signature characterized by tones at the blade passage frequency as well as broadband noise. Fan noise reductions have been achieved by the installation of passive acoustic liners in both the nacelle inlet and aft ducts to attenuate tonal and broadband components. The nacelle adds weight, which increases specific fuel consumption, and thus the designer must reconcile two conflicting criteria, maximizing liner treatment for noise control and minimizing nacelle size. It is critical to develop a liner design that provides a targeted noise reduction spectrum by making the most effective use of available real estate. This, in turn, requires greater knowledge of how noise propagates through the duct than is currently available. One purpose of the Curved Duct Test Rig (CDTR) is to provide a test-bed for generation and propagation of sound in a large

\footnotetext{
* Senior Research Scientist, Aeroacoustics Branch, (757) 864-5279, email: carl.h.gerhold@nasa.gov, Associate Fellow, AIAA

${ }^{\dagger}$ Senior Research Scientist, Structural Acoustics Branch, phone: (757) 864-5272, email: michael.g.jones@nasa.gov, Associate Fellow, AIAA

* Aerospace Engineer, Aeroacoustics Branch, phone: (757) 864-6277, email: martha.c.brown@nasa.gov

$\S$ Aerospace Engineer, Structural Acoustics Branch, phone: (757) 864-5784, email:

d.m.nark@nasa.gov, Member, AIAA
} 
scale duct, representative of the aft bypass duct, and for evaluation of noise control methodologies. This report documents the evaluation of one such industry-designed passive liner concept.

Computational models can be used to aid in the process of evaluating liner designs, and are particularly useful in testing candidate designs and selecting promising technologies for experimental verification. The model must first be calibrated to ensure that it provides accurate representation of the sound propagation in the duct. Thus another purpose of the CDTR is validation of a computational model that has been developed separately to estimate sound propagation in the duct. This report documents the process of validation of the model using measured results for acoustic performance of an industry-designed passive liner sample.

The liner sample was evaluated in the Curved Duct Test Rig at no flow and the results were shown in a previous report [1]. Flow paths with and without curvature in the propagation direction were evaluated. It was found that, when one side of the duct is treated with the liner and the opposite wall is acoustically hard, the asymmetry of treatment caused the transverse component of the mode of the incoming sound to scatter into a less attenuated cut-on mode. This scattered energy degraded the total attenuation when all the modal components were summed. For the liner used in that study, no significant difference in the acoustic performance of the liner was found between the straight and the curved configurations. When both sides of the duct were treated with the liner, the total attenuation increased significantly because no appreciable mode scattering occurred. The peak amplitude in the attenuation spectrum decreased as the curvature increased, and this trend was found to occur for all recorded incident modes. It was hypothesized that this unexpected trend was due to the fact that, at no flow, the liner was operating well away from its design point; and that, when the liner is used at conditions closer to those for which it was designed, it would perform in a more predictable way. The liner parameters were chosen to maximize attenuation with flow and this report summarizes experiments for an average Mach number of 0.275 in the duct.

\section{Description of the Test}

\section{A. Curved Duct Test Rig (CDTR)}

Figure 1 is a photograph of the Curved Duct Test Rig. A brief description of the test rig is presented here, and more details are found in previous papers by the authors [2,3]. Air is supplied to the test rig from a centralized air supply and passes through a set of mufflers (not shown in the photograph), an elbow with turning vanes, and a flow conditioning section to smooth the air flow and reduce turbulence. A transition section reduces the duct cross section from $76 \mathrm{~cm} \times 76 \mathrm{~cm}$ (30 in $\times 30$ in) to the test section, which is $15 \mathrm{~cm}$ x $38 \mathrm{~cm}$ (6 in x 15 in). Downstream of the test section is a diffuser that reduces the air flow speed and provides a near-anechoic termination. The test section consists of 6 duct pieces: the source, two measurement, and two filler sections, and the liner test section. The source section contains an array of 16 loudspeakers that are tightly coupled to the duct ports $(8.00 \times 1.91 \mathrm{~cm})$ via rigid connecting tubes ranging in length from $27.94 \mathrm{~cm}(11.00$ in) to $31.12 \mathrm{~cm}(12.25$ in). The ports are co-located in two planes and positioned to maximize acoustic energy transfer to all cut-on modes. The ports contain a porous honeycomb to inhibit flow turbulence. There are two measurement sections with microphones, one upstream of the liner test section and one downstream. The microphone arrays are identical in both sections, containing 47 flush-mounted B\&K model 4951, $6.35 \mathrm{~mm}(0.25 \mathrm{in})$ piezoceramic microphones. Selection of the microphone locations to maximize resolution of the modal decomposition is discussed in reference 2. A subarray consisting of 31 of the microphones in the upstream array is used to measure the error signal for the control system that generates the sound in the duct. The purpose of the control system is to isolate a targeted mode in the duct at a user-specified amplitude at the inlet to the liner test section. The liner test section contains the liner under investigation. The top and bottom walls of the liner test section are $12.7 \mathrm{~mm}(0.5 \mathrm{in})$ thick aluminum plate and the liner samples form the side walls. Figure 2 shows the straight flow path with liner samples in place on both side walls of the liner test section. Each liner sample is $38 \mathrm{~cm}$ (15 inch) high and (nominally) $81 \mathrm{~cm}$ (32 inch) long in the flow direction. The space behind the liner sample is filled with a rigid foam block that is used to hold the liner samples in place. A thin non-rigid foam is used to fill in any voids between the liner and the rigid foam block. Through bolts affix the liner sample firmly against the rigid foam and the wall of the liner test section. Finally, two $20 \mathrm{~cm}(8 \mathrm{inch})$ long 
rigid wall duct sections are on either end of the liner test section. Their purpose is to separate the microphones from the near field effects due to sudden impedance discontinuities at either end of the liner.

\section{B. Liner Samples}

The liners consist of an impervious $1.02 \mathrm{~mm}(0.040$ inch) thick backing sheet, $19.05 \mathrm{~mm}(0.75$-inch) thick honeycomb core, and $0.64 \mathrm{~mm}(0.025 \mathrm{inch})$ thick perforate cover sheet. The perforate is $8.7 \%$ open area with $0.99 \mathrm{~mm}(0.039$-inch) diameter holes. The liner materials are representative of single degree of freedom liners in use in engines and the liner is designed to achieve a targeted impedance in the presence of grazing flow ranging in Mach number from 0.3 to 0.5 . The resonance frequency of the liner installed in the CDTR occurs in the middle of the operating range of the CDTR, between 300 and $2400 \mathrm{~Hz}$.

In addition to the straight path, two curved path configurations were fabricated. The exit flange in the first configuration is offset from the entrance by $1 / 2$ duct dimension $(76 \mathrm{~mm})$ and in the second configuration the offset is 1 duct dimension $(152 \mathrm{~mm})$. The liner test section with the 1D offset liner sample installed is shown in Figure 3.

The liners are designed so that the slope increases linearly from zero at the inlet to a maximum at the midpoint, then decreases linearly from the midpoint to a zero slope at the discharge. The radius of curvature is $17 \mathrm{~cm}$ (44 inch) for the 1D offset and $34 \mathrm{~cm}$ ( 86 inch) for the $1 / 2 \mathrm{D}$ offset. Hard wall equivalents of the liner samples were fabricated using the same design parameters, but with the perforate cover sheet replaced with a solid sheet. The straight and curved liner samples were fabricated by Goodrich Aerostructures, and hard wall versions were fabricated in-house using the same design.

Various configurations of hard and treated walls were evaluated. The liner sample configurations are identified by the designation Cabcd where $\mathrm{C}$ denotes configuration and the terms $\mathrm{a}, \mathrm{b}, \mathrm{c}$, and $\mathrm{d}$ are defined:

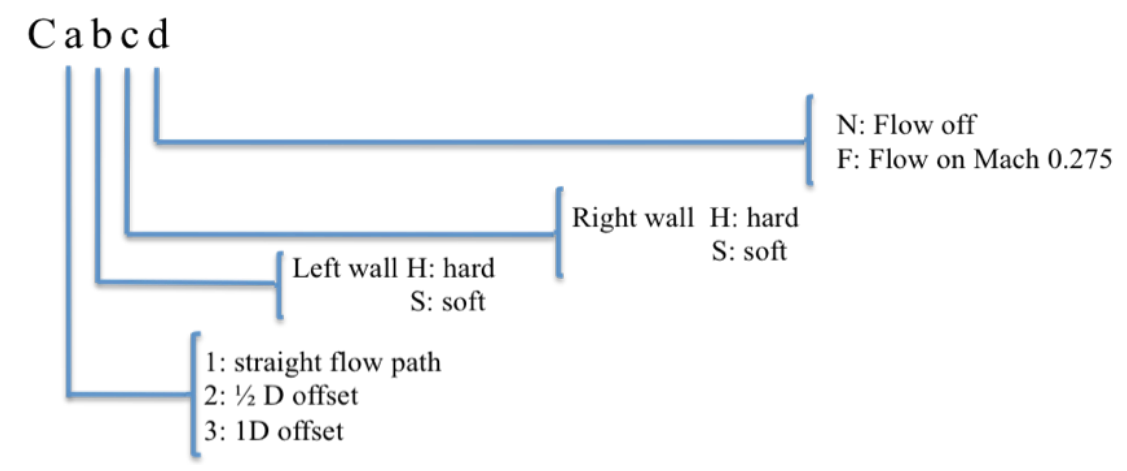

The configurations tested are summarized in Table I. Note that no data were acquired for three conditions; straight flow path, mix of treated and hard wall, flow on at Mach 0.275 and for curved, $1 / 2 \mathrm{D}$ offset, soft wall both sides, flow on at Mach 0.275 . The data reported in the current paper are for the straight $(\mathrm{C} 1)$ and $1 \mathrm{D}$ offset (C3) configurations. 


\begin{tabular}{|l|l|c|c|}
\hline \multirow{2}{*}{ Flow Path } & \multicolumn{2}{|c|}{ Liner Description } & \multicolumn{2}{c|}{ Flow } \\
\cline { 2 - 4 } & & $\mathbf{M = 0 . 0 0 0}$ & $\mathbf{M = 0 . 2 7 5}$ \\
\hline Straight & Both wall treated & C1SSN & C1SSF \\
\hline Straight & Right wall treated, left wall hard & C1HSN & X \\
\hline Straight & Left wall treated, right wall hard & C1SHN & X \\
\hline Straight & Both wall hard & C1HHN & C1HHF \\
\hline 1/2-D offset & Both wall treated & C2SSN & X \\
\hline 1-D offset & Both wall treated & C3SSN & C3SSF \\
\hline 1-D offset & Right wall treated, left wall hard & C3HSN & C3HSF \\
\hline 1-D offset & Left wall treated, right wall hard & C3SHN & C3SHF \\
\hline 1-D offset & Both wall hard & C3HHN & C3HHF \\
\hline
\end{tabular}

Table I. Liner sample testing configurations, $X$ indicates no data acquired

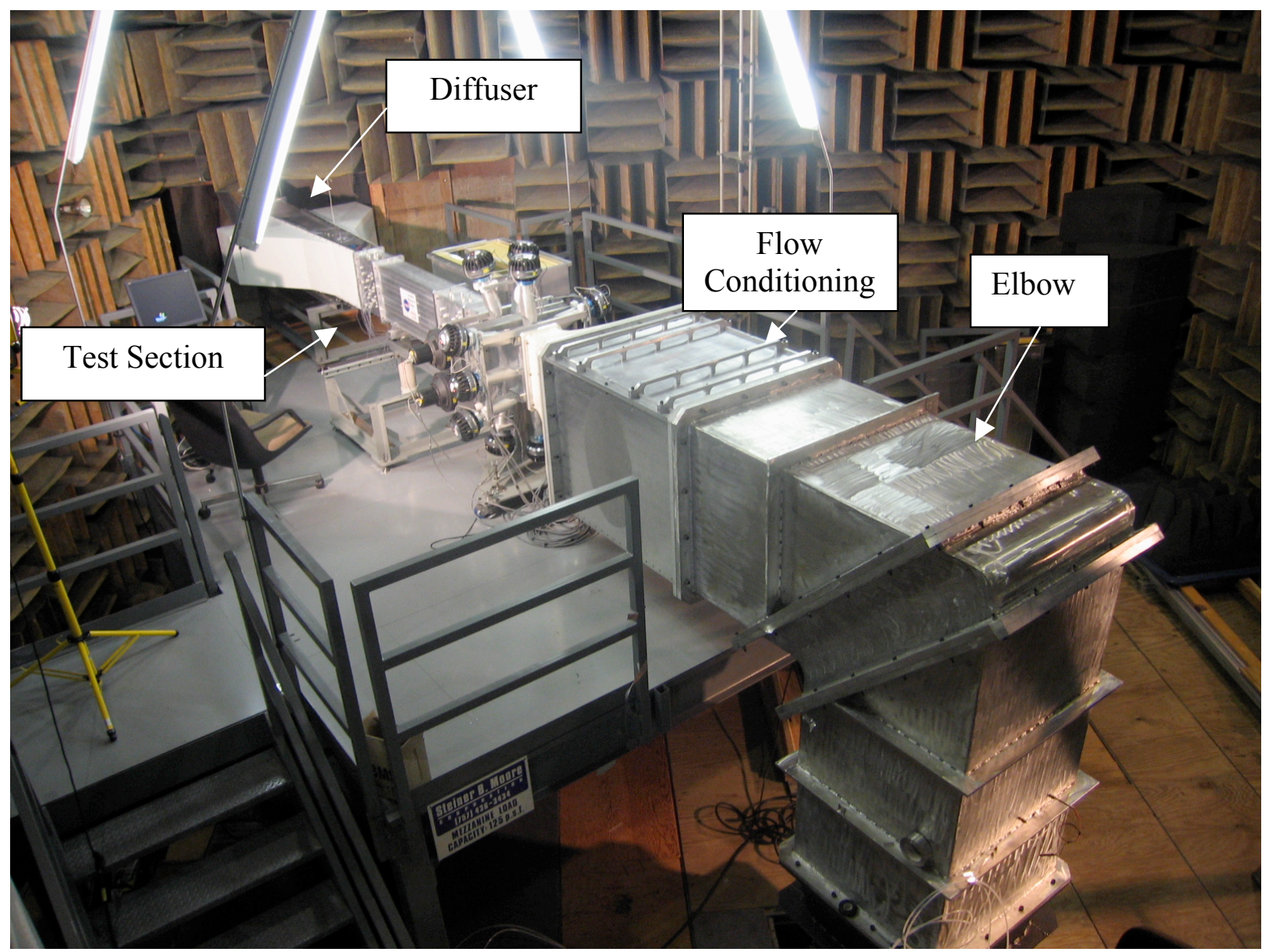

Figure 1. Curved Duct Test Rig in the Anechoic Noise Research Facility. 


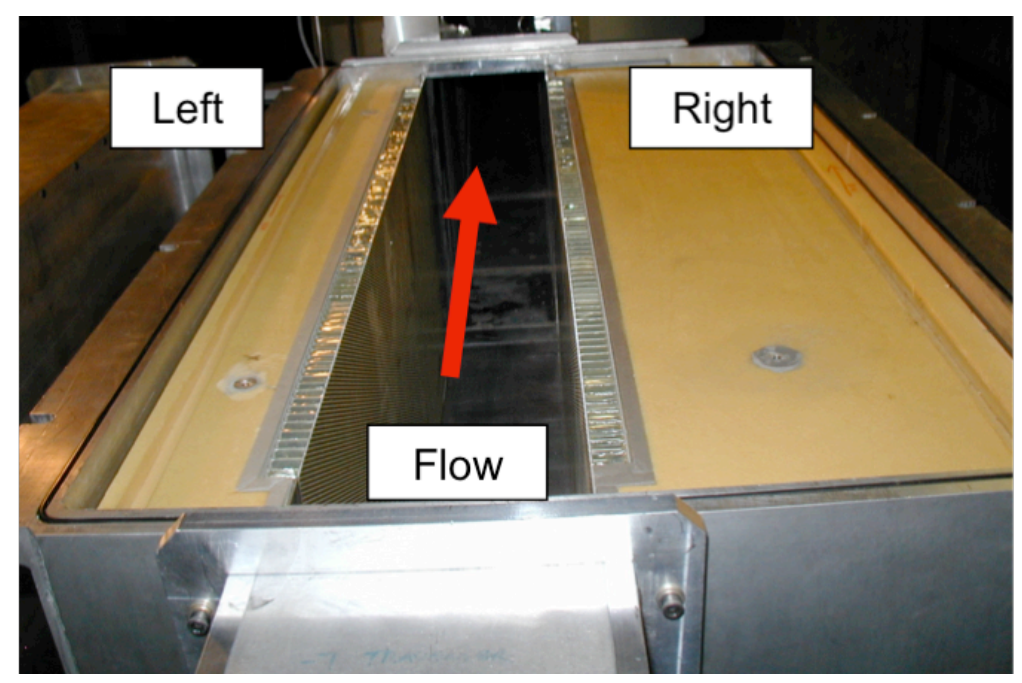

Figure 2. Liner test section with straight liner samples on both walls, top removed, view looking downstream.

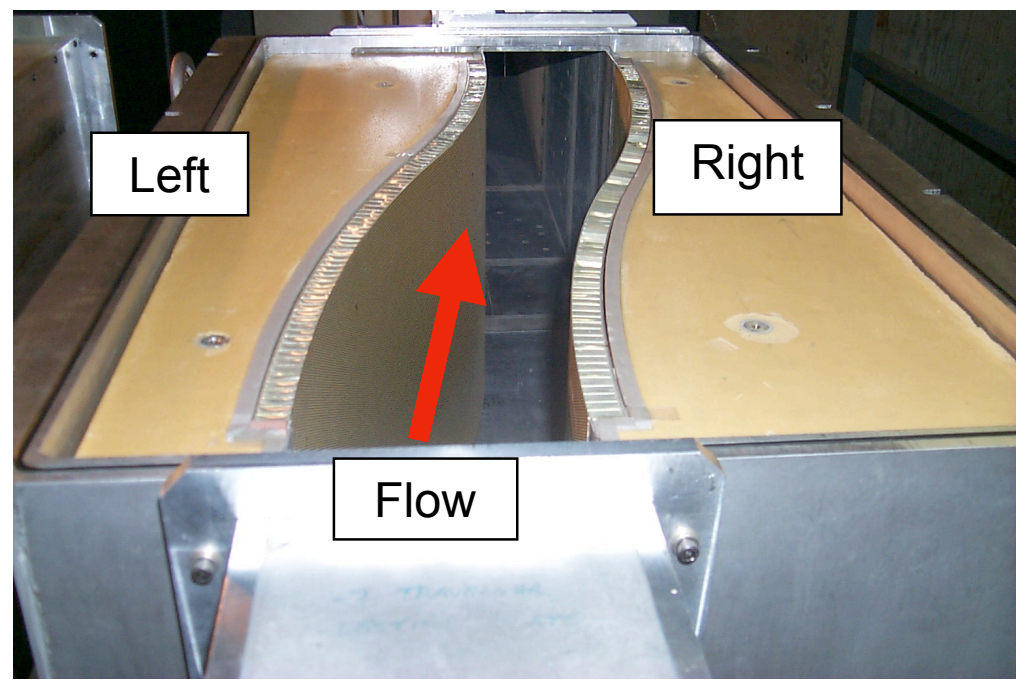

Figure 3. 1D offset curved liner sample in the liner test section of the CDTR, top removed, view looking downstream.

Tonal acoustic excitation, over the frequency range 300 to $2400 \mathrm{~Hz}$, is generated upstream of the liner test section. The sound is controlled such that a user-specified mode shape propagates in the duct upstream of the liner. Vertical mode orders ranging from 0 to 5 , and horizontal mode orders from 0 to 2 can be isolated by the control system. The controlled mode is generally at least $10 \mathrm{~dB}$ higher than any other cut-on mode in the duct. The system is capable of operating with flow, the maximum condition of which is uniform flow outside the boundary layer through the liner test section at Mach 0.275 . Table II shows the frequencies at which the horizontal (n) and vertical (m) modes are expected to cut on in the duct at standard conditions. Horizontal modes in the CDTR correspond to radial modes in the engine bypass duct and vertical modes in the CDTR correspond to circumferential modes. The cut-on frequencies for no flow are the first set of numbers and the cut-on frequencies when uniform flow is present in the duct at Mach 0.275 are shown in parentheses. 


\begin{tabular}{|c|c|c|c|}
\hline n (horiz) & $\mathbf{0}$ & $\mathbf{1}$ & $\mathbf{2}$ \\
\hline m (vert) & 0 & $1128(1084)$ & $2256(2168)$ \\
\hline 0 & $451(434)$ & $1215(1168)$ & $2301(2211)$ \\
\hline 1 & $902(867)$ & $1445(1388)$ & $2430(2335)$ \\
\hline 2 & $1354(1301)$ & $1762(1693)$ & \\
\hline 3 & $1805(1734)$ & $2128(2045)$ & \\
\hline 4 & $2256(2168)$ & & \\
\hline 5 & & & \\
\hline
\end{tabular}

Table II. Cut-on frequencies (in $\mathrm{Hz}$ ) of modes in the $15.2 \mathrm{~cm}$ (horizontal) $\mathrm{x} 38.1 \mathrm{~cm}$ (vertical) duct at standard conditions. Values without parentheses are at Mach 0.0 , values within parentheses are with flow at Mach 0.275

\section{Results}

Experimental results are discussed in the first two sections in which configuration effects are compared. In the first case, attenuation for the left side treated, right side hard (C3SHF) is compared with the right side treated, left side hard (C3HSF) of the 1D offset configuration. In the second case, the 1D offset flow path (C3SSF) is compared to the straight flow path (C1SSF) for both sides treated. Next, the computational model is used to estimate the sound propagation in the lined duct with flow is introduced, and is validated by comparison with experimental results for both sides treated. Finally, a computational study is undertaken to determine the liner design parameters that provide optimum attenuation in the frequency range from 1900-2400 Hz. In all instances, the average Mach number in the duct is 0.275.

While sound in a specific mode is input to the liner test section, the configuration may alter the modal distribution downstream of the liner. The acoustic power balance was introduced in a previous paper by the authors [1] as a tool to quantify the modal redistribution. The power balance is the modal distribution of the power downstream of the liner test section normalized by the total power upstream. It is intended to identify the attenuation and scattering of the incident mode. It was demonstrated previously that the incident mode dominates upstream of the liner test section. Thus, any value of the incident mode power downstream that is less than 1.0 indicates attenuation of the incident mode, and any increase in power of any other mode indicates that energy has been scattered into it from the incident mode. In the section to follow, when the term total attenuation is used, it is taken to mean the attenuation of the sum of all the modes in the duct.

\section{A. Comparison of Experimental Results: One side of Duct Treated}

Figure 4 shows the total attenuation for the $(0,0)$ mode incident on the liner test section, and it compares the right side treated (C3HSF) and left side treated (C3SHF) cases. The left side treated configuration gives more attenuation throughout most of the frequency range. Figures 5 and 6 are the acoustic power balance in the duct for the $(0,0)$ mode incident on configurations C3HSF and C3SHF, respectively. It is seen from Figures 5 and 6 that the attenuation of the incident mode, $(0,0)$, is similar whether the right side or the left side is treated. However, more energy is scattered into the next transverse mode, $(0,1)$, when the right side is treated (Figure 5) than when the treatment is on the left side (Figure 6). Thus, the total attenuation is less for the configuration in which the right side is treated. This is observed in Figure 4. 


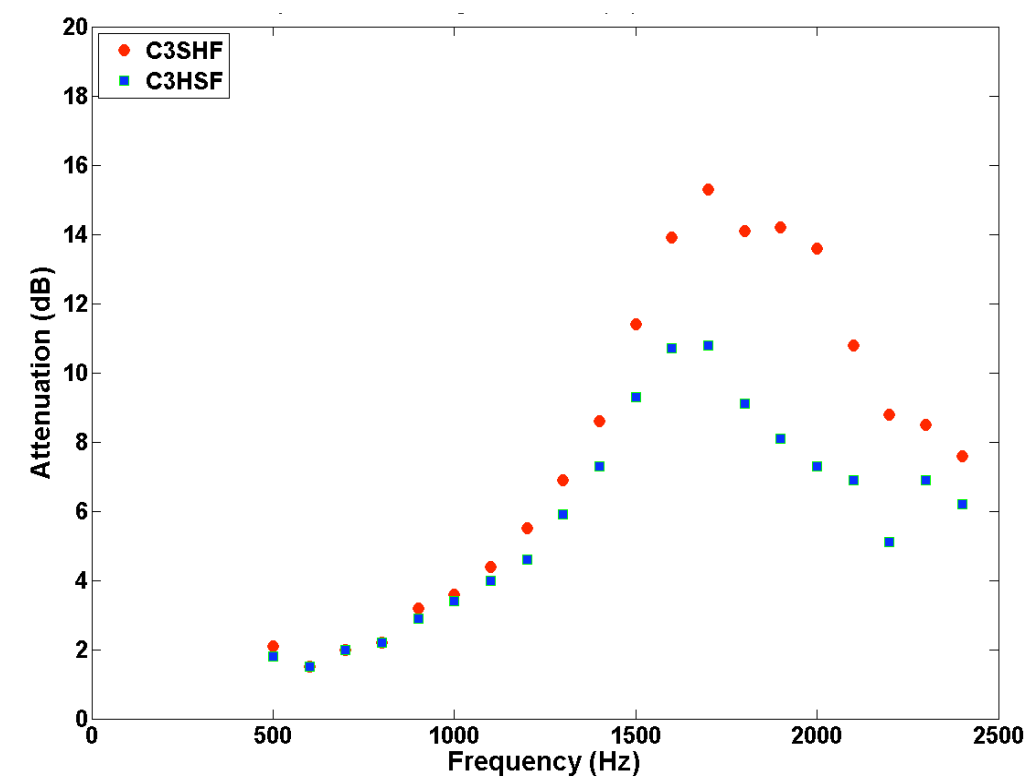

Figure 4. Measured total attenuation, $(0,0)$ mode incident, mach $0.275,1 \mathrm{~d}$ offset, liner treatment on one side of duct. Compares left side (C3SHF) to right side (C3HSF) treated.

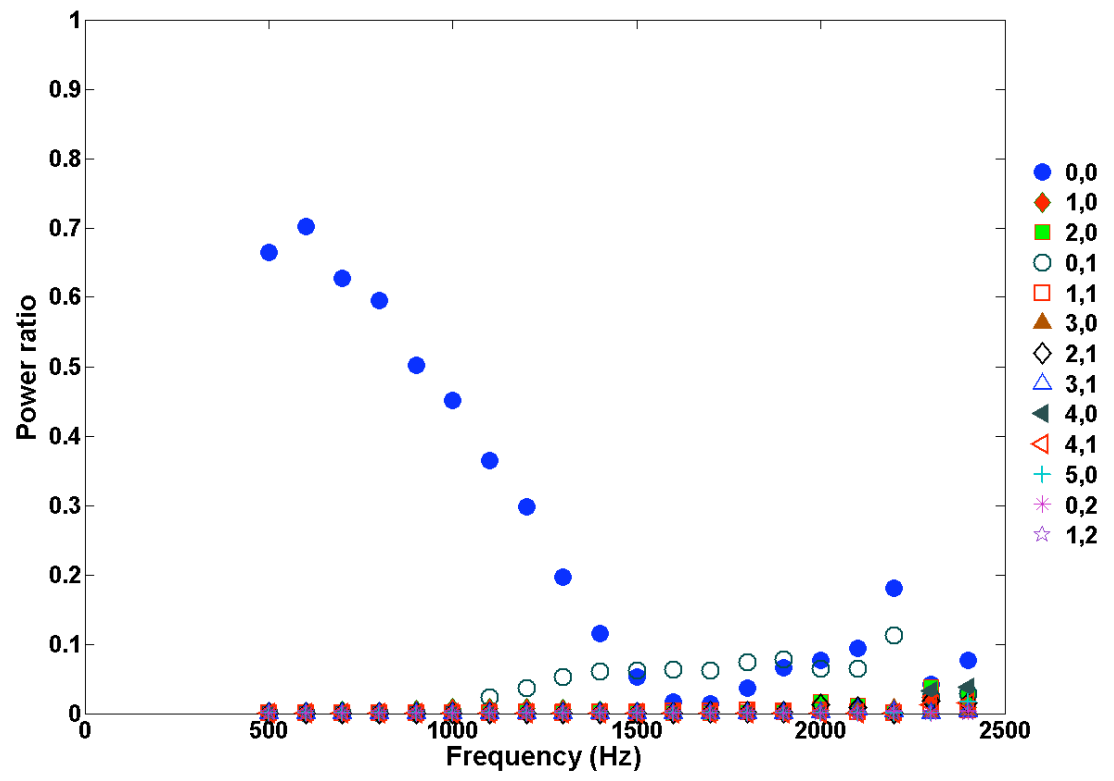

Figure 5. Measured power balance, $(0,0)$ mode incident, Mach 0.275, 1D offset, configuration C3HSF. 


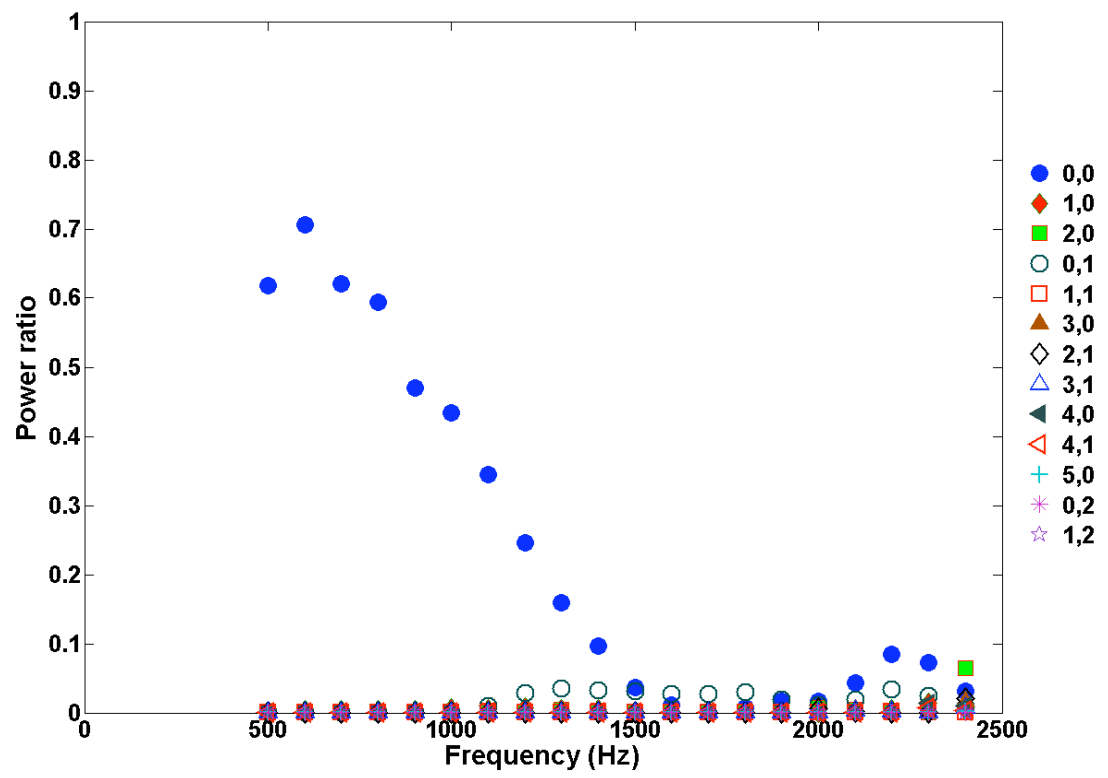

Figure 6. Measured power balance, $(0,0)$ mode incident, Mach 0.275, $1 \mathrm{D}$ offset, configuration C3SHF.

Figure 7 shows that the total attenuation for the $(2,0)$ mode incident is also greater for the configuration in which the left side is treated than it is for the right side treated. The comparative mode distributions, not shown here, indicate that energy is scattered into the $(2,1)$ mode from the $(2,0)$ mode incident in both cases, but it appears that more energy scatters into the higher transverse mode for the case in which the treatment is on the right side. This result is consistent with the $(0,0)$ incident mode results. The comparative mode distribution plots are included in reference 4.

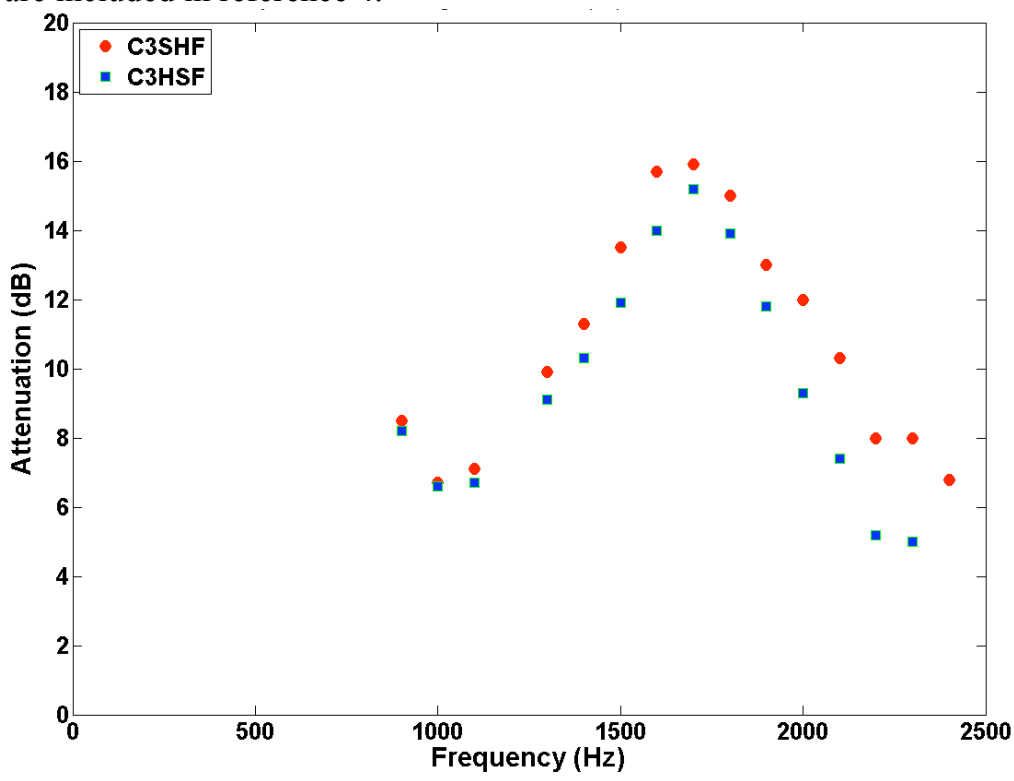

Figure 7. Measured total attenuation, $(2,0)$ mode incident, Mach 0.275 , $1 \mathrm{D}$ offset, liner treatment on one side of duct. Compares left side (C3SHF) to right side (C3HSF) treated.

Figure 8 shows the measured total attenuation for the $(0,1)$ mode incident on the liner configuration with one side treated. When the treatment is on the right side, the peak of the attenuation curve occurs at 1600 Hz. When the left side is treated, the peak occurs at $2100 \mathrm{~Hz}$. Consistent with the observation for the $(0,0)$ and $(2,0)$ modes incident, the left side treated attenuation is greater than right side treated attenuation at 
higher frequencies. The left side treated case attenuation is much less than the right side just at cut-on, 1200 $\mathrm{Hz}$.

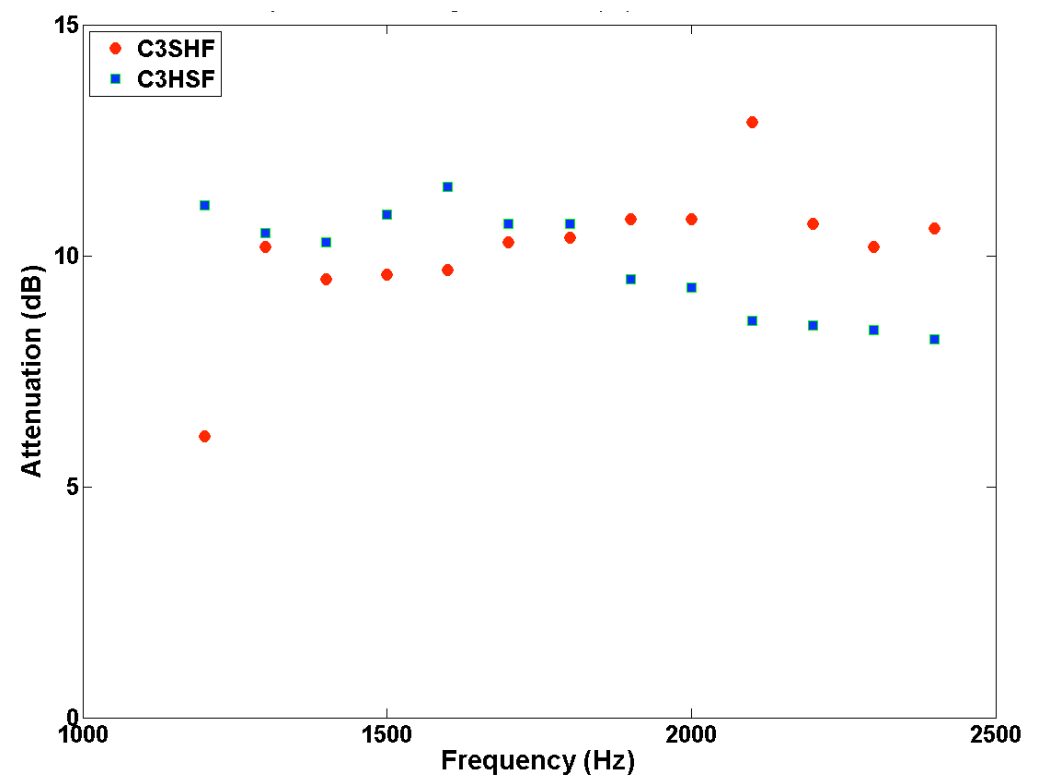

Figure 8. Measured total attenuation, $(0,1)$ mode incident, Mach 0.275 , 1D offset, liner treatment on one side of duct. Compares left side (C3SHF) to right side (C3HSF) treated.

The mode distributions for these two cases are shown in Figure 9, C3HSF and Figure 10, C3SHF. The next higher transverse mode, $(0,2)$, does not cut on until $2260 \mathrm{~Hz}$. Thus, the higher order mode into which the $(0,1)$ mode could scatter energy is cut off for much of the measurement spectrum. The $(0,1)$ mode scatters instead into the lower $(0,0)$ mode. In fact the $(0,0)$ mode becomes dominant downstream above $1900 \mathrm{~Hz}$ for the right side treated and above $1450 \mathrm{~Hz}$ for the left side treated. Thus, the total attenuation for the left side treated case at higher frequencies shown in figure 8 is controlled by the $(0,0)$ mode. The mode distributions for both cases show a dip in the power curve at $2100 \mathrm{~Hz}$, but the decrease of power is greater for the left side treated case. This is reflected in the higher attenuation at $2100 \mathrm{~Hz}$ for the left side treated in Figure 8 .

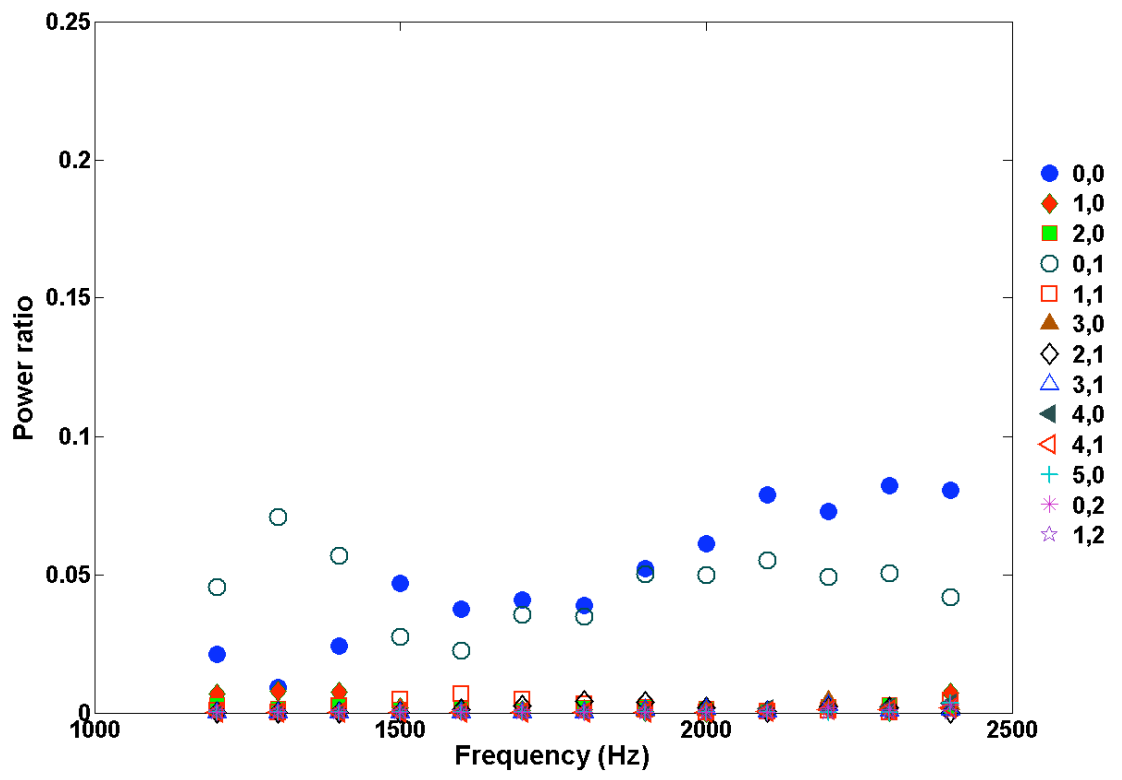

Figure 9. Measured power balance, $(0,1)$ mode incident, Mach 0.275, 1D offset, configuration C3HSF. 
The contribution of the $(1,0)$ mode is unusually strong for the left side treated case just above cut-on of the $(0,1)$ mode, at $1200 \mathrm{~Hz}$. This contribution is what causes the total attenuation of the left side treated case to be $5 \mathrm{~dB}$ less than the right-side treated attenuation at $1200 \mathrm{~Hz}$, as shown in Figure 8.

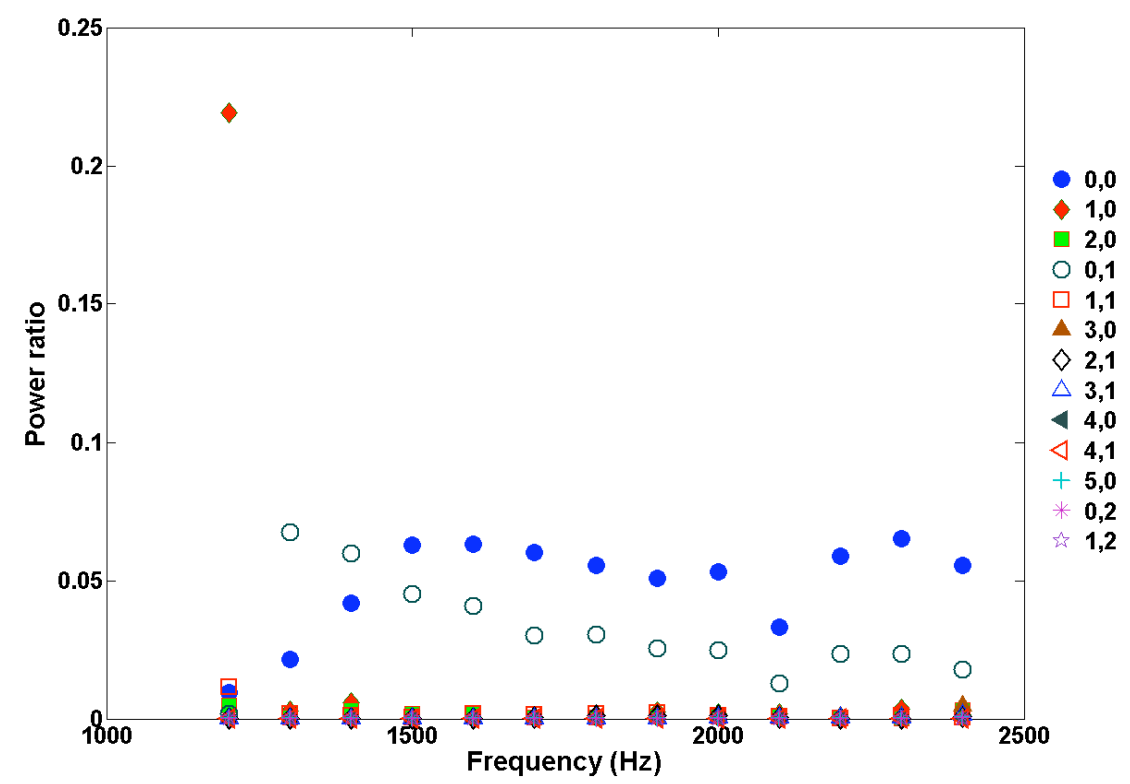

Figure 10. Measured power balance, $(0,1)$ mode incident, Mach 0.275, 1D offset, configuration C3SHF.

\section{B. Comparison of Experimental Results: Both Sides of Duct Treated}

Figure 11 shows the measured total attenuation of the $(0,0)$ mode incident in the duct with flow on at Mach 0.275, with liners on both sides of the duct, for the straight (C1SSF) and 1D offset curved (C3SSF) liner configurations. The curved duct is seen to provide higher attenuation than the straight duct at all frequencies except the frequency of peak attenuation.

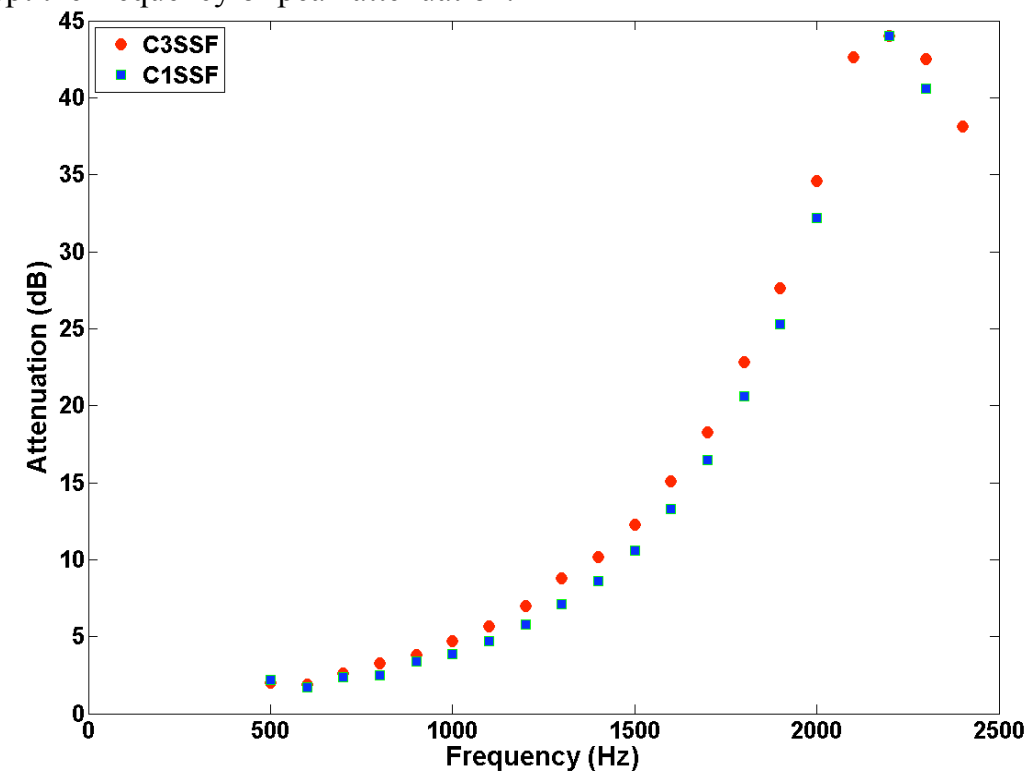

Figure 11. Total attenuation, $(0,0)$ mode incident, Mach 0.275 , liner treatment on both sides of duct. Compares straight (C1SSF) to $1 \mathrm{D}$ offset (C3SSF) 
Figures 12 and 13 show the attenuation with the $(2,0)$ and $(0,1)$ mode incident, respectively. The attenuation by the curved liner is slightly higher than that for the straight liner, except at the frequency of peak attenuation, with the $(2,0)$ mode incident, as is shown in Figure 12. The attenuations for the straight and offset liner configurations are roughly equivalent for the $(0,1)$ mode incident, as can be seen in Figure 13.

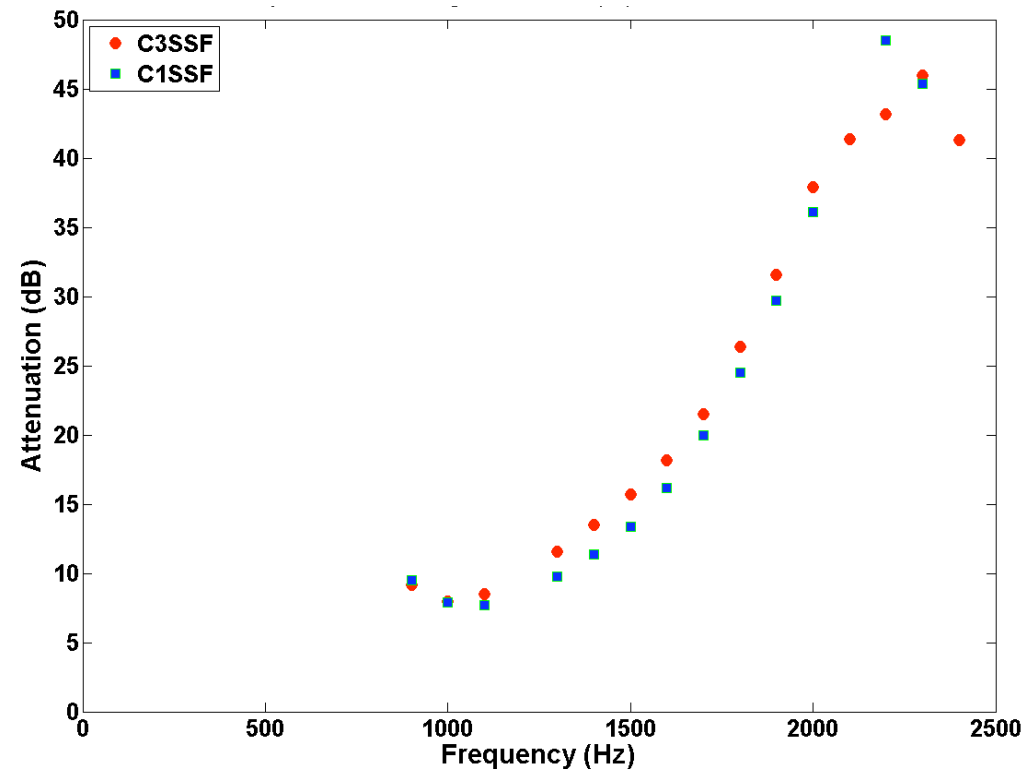

Figure 12. Measured total attenuation, $(2,0)$ mode incident, Mach 0.275 , liner treatment on both sides of duct. Compares straight (C1SSF) to $1 \mathrm{D}$ offset (C3SSF)

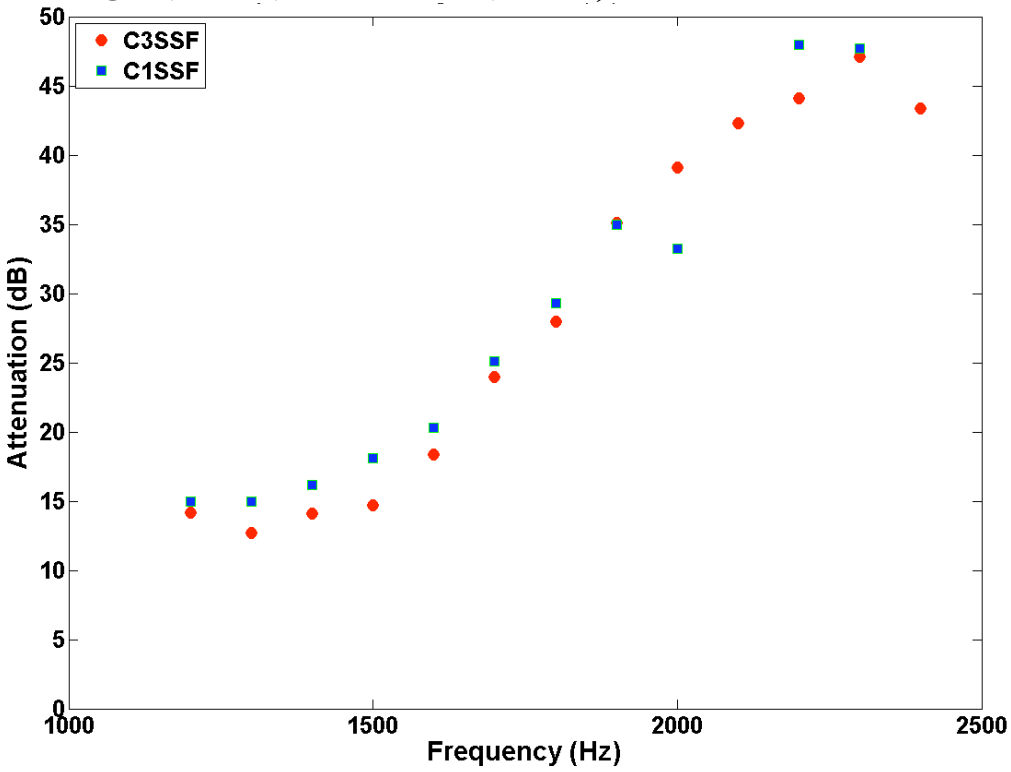

Figure 13. Measured total attenuation, $(0,1)$ mode incident, Mach 0.275, liner treatment on both sides of duct. Compares straight (C1SSF) to 1 D offset (C3SSF).

\section{Computational Model}

The experimental results are compared to attenuations calculated by a numerical code that computes the sound propagation in a lined duct. The code, designated CH3DPA, is based on convected Helmholtz 
equations, for 3D solutions, solved using a parabolic approximation. It is based on the CDUCT code developed by Robert Dougherty [5], currently of Optinav. The code has been extensively modified and refined by a team led by Doug Nark of NASA Langley Research Center. The designation CH3DPA replaces an earlier designation CDUCT-LaRC and is part of a standardization of naming convention for the various computer codes in use at NASA Langley Research Center. The code is very efficient, which makes well suited for parametric studies of liner design. A more complete discussion of the operation of CH3DPA as it relates to the CDTR may be found in reference 1. CH3DPA uses as input the acoustic pressure distribution in the hard wall duct upstream of the liner test section and the description of the liner treatment. The sound pressure is described in terms of the amplitude and phase of each of the cut-on modes in the upstream section and is based on the data measured in the CDTR. The liner description is input in terms of the spectrum of wall impedance.

The impedance model used by CH3DPA is the Two Parameter Impedance Prediction model. This model was discussed in a paper presented to the AIAA Aeroacoustics Conference in 2008, by Jones, et al [6]. The model defines the impedance parameters, the resistance and reactance, of a single-degree-of-freedom perforate over honeycomb core liner. The expression for the impedance, normalized by the characteristic impedance, $\rho c$, is given as:

$Z=\theta+i \chi$

where $\theta$ is the resistance and $\chi$ is the reactance. The formulae for the reactance and resistance are shown in Appendix A. The dependence of the impedance on physical parameters of the liner, such as core depth, perforate sheet thickness and percent open area, and hole diameter is shown.

Since the impedance of the liner sample used in the CDTR for this series of experiments is not known explicitly, it is estimated from measurements performed in the Grazing Incidence Tube (GIT) on a similar liner, using an eduction method discussed in a paper by Jones, et al [7]. The GIT liner and the CDTR liner both have the same perforate and honeycomb core cell cross-section. The honeycomb core depth of the GIT liner sample is $38.2 \mathrm{~mm}$ (1.5-inch), where the core depth of the CDTR liner, is $19.1 \mathrm{~mm}(0.75$ inch). The flow speed in the duct and the Sound Pressure Level are similar for both liners. It can be seen from the expression for the resistance in Appendix A that, since the perforate and the test conditions are the same in both cases, the resistance of the CDTR liner can be assumed to be the same as the resistance of the GIT liner.

Appendix A shows that the reactance component of the impedance consists of two parts; a mass reactance, which depends largely on the perforate thickness and hole configuration, and a part that depends on the core depth. The mass reactance is assumed to be the same for both liners, since the perforate is the same. The reactance of the current liner is estimated from:

$\chi_{0.75}=\chi_{1.5}+\cot \left(k * L_{1.5}\right)-\cot \left(k * L_{0.75}\right)$

where $\chi_{0.75}$ is the reactance of the 0.75 inch liner, $\chi_{1.50}$ is the reactance of the 1.50 inch liner, $\mathrm{L}_{0.75}$ is the core depth of the 0.75 inch liner, $\mathrm{L}_{1.50}$ is the core depth of the 1.5 inch liner, and $\mathrm{k}$ is the wavenumber. 


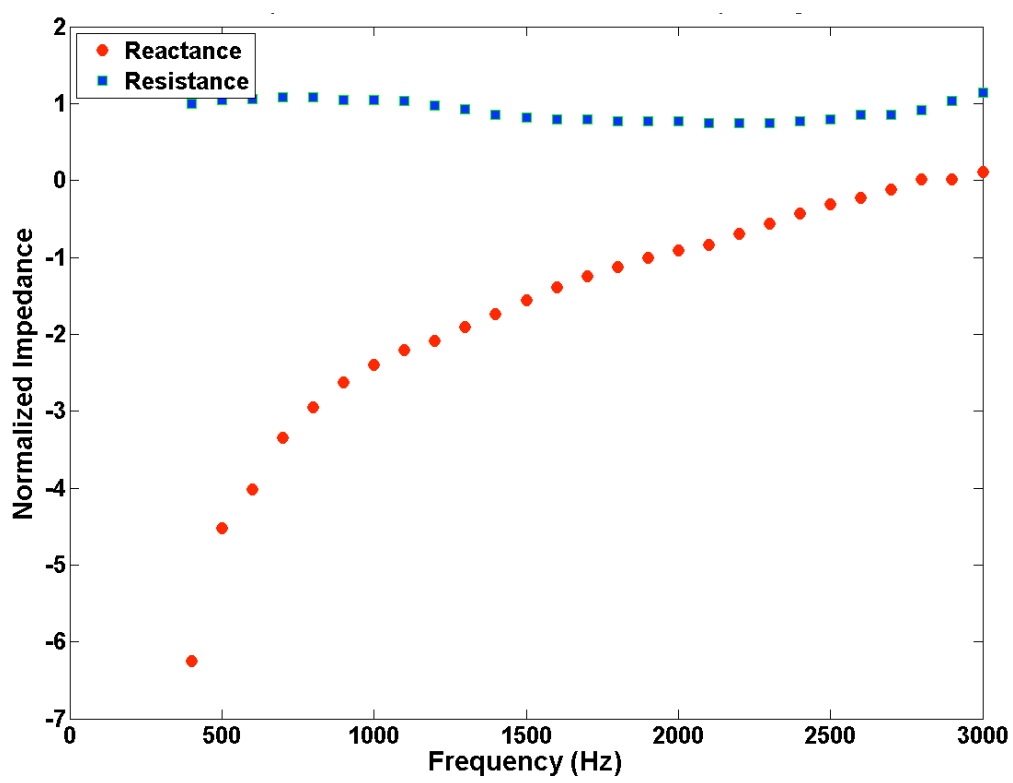

Figure 14. Impedance of the liner sample based on impedance educed in GIT at Mach 0.3 and $130 \mathrm{~dB}$

Figure 14 shows the estimated impedance of the CDTR liner sample based on the impedance of the GIT liner.

\section{Comparison of Experimental to Computational Results}

Comparison between measured and computed attenuations with the $(0,0)$ mode incident for the straight liner, both sides treated, configuration C1SSF, is shown in Figure 15.

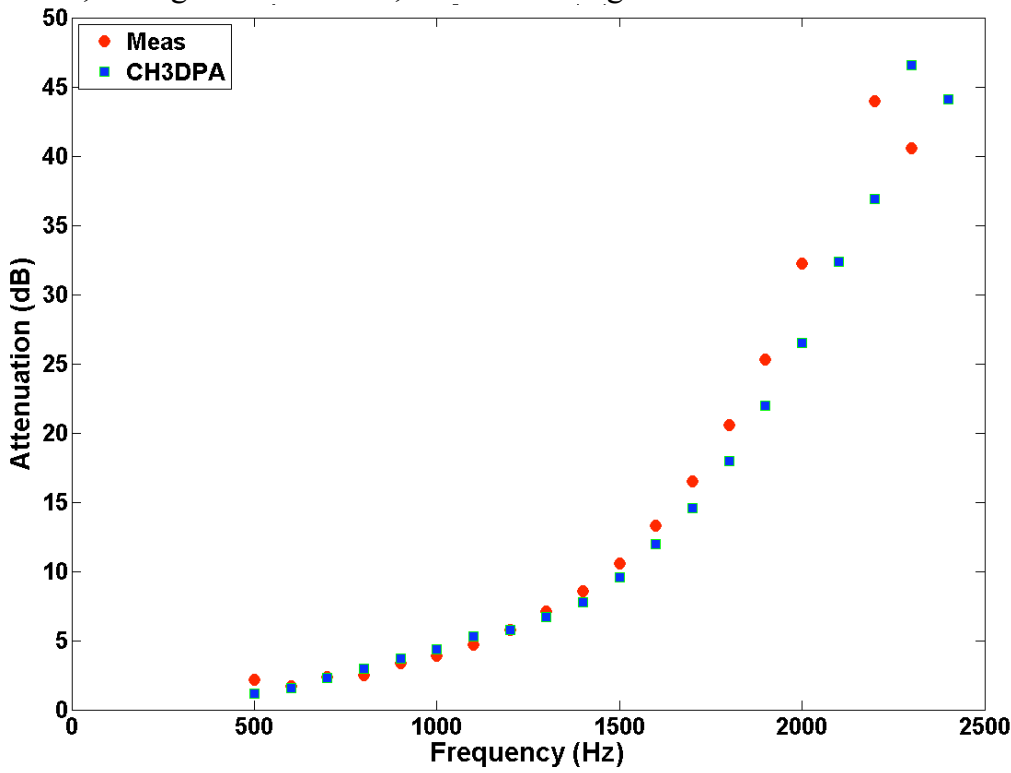

Figure 15. Measured total attenuation, $(0,0)$ mode incident, Mach 0.275 , straight liner treatment on both sides of duct. Compares measured data to CH3DPA calculation.

The computation follows the measured data closely for the $(0,0)$ mode incident case for frequencies below $1500 \mathrm{~Hz}$, as is seen in Figure 15. Although a data point is missing in the measurement, it appears that the measured attenuation peaks at $2200 \mathrm{~Hz}$ where the computed attenuation peaks at $2300 \mathrm{~Hz}$. The energy 
average error is evaluated as a way to assess the closeness of fit between the measured and computed attenuations. It is evaluated from:

error $=-10 * \log _{10}\left[\frac{\sum_{n=1}^{N} 10^{\left(-\Delta_{n} / 10\right)}}{N}\right]$

where $\Delta_{\mathrm{n}}$ is the difference between measured and computed attenuation at $\left(\mathrm{f}_{\mathrm{n}}\right)$ and $\mathrm{N}$ is the number of frequency bins.

The energy average error for frequencies from 1900-2400 Hz, is $5.6 \mathrm{~dB}$. It was also observed that the computed attenuation follows the measured attenuation but peaks at $100 \mathrm{~Hz}$ past the measured peak for the $(2,0)$ and $(0,1)$ modes incident. These curves are included in reference 4.

The differences between the measured and computed attenuations are thought to be due to inaccuracy in the values of the parameters used in the impedance. Since the magnitudes of the peak attenuation are similar, it appears that the resistance component of the impedance is correctly modeled. The shift in frequency can be attributed to the length-dependent part of the reactive component of the impedance. This is the part that was modified in equation (2) to account for the difference between the GIT liner sample and the CDTR liner sample. If the measurement of the core depth of either of the two liner samples is in error, this would shift the kL-dependent component of the reactance curve. A sensitivity study was undertaken to determine what order of magnitude of length measurement is required to resolve the $100 \mathrm{~Hz}$ discrepancy between the measured and computed attenuations. The impedance was recalculated for a matrix of conditions in which the core depth for either the GIT or the CDTR liner could be the nominal value, the nominal value plus a delta, or the value minus a delta. The attenuation was calculated from 2000 to $2400 \mathrm{~Hz}$. It was found that a delta of $1.59 \mathrm{~mm}(1 / 16 \mathrm{inch})$ causes the peak frequency of the attenuation curve to shift toward $2200 \mathrm{~Hz}$. Figure 16 shows the new impedance curve.

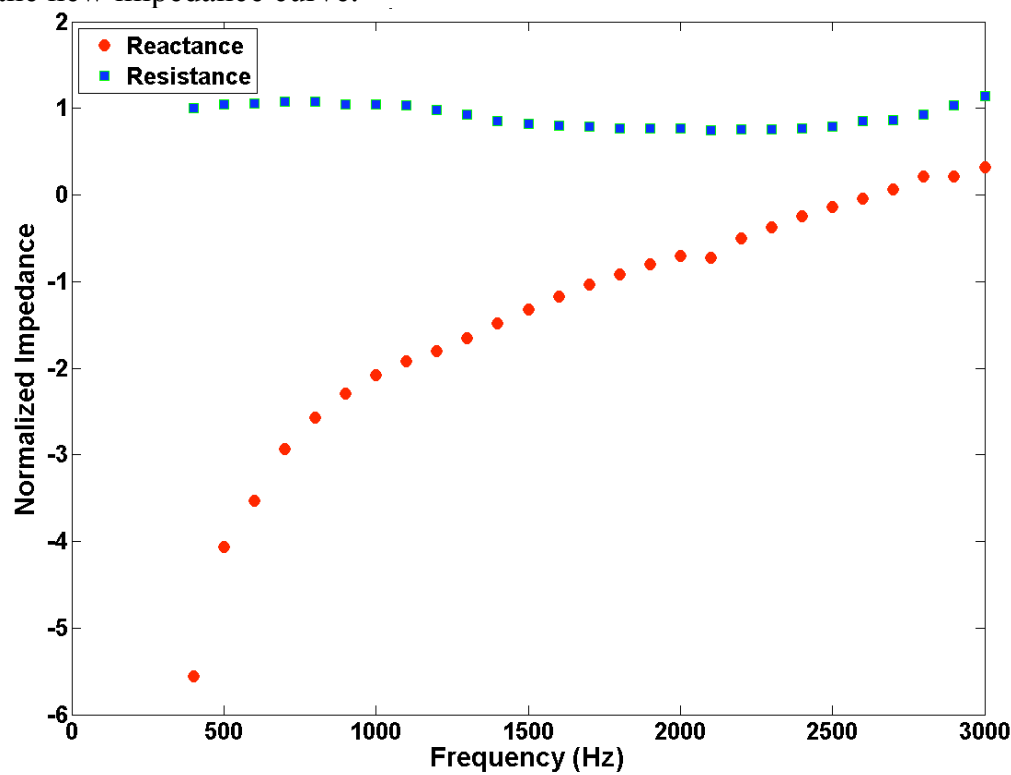

Figure 16. Impedance of 0.75 inch liner with length adjustment to match measured peak frequency.

Comparison between measured and computed attenuations with the $(0,0)$ mode incident on the straight liner, both sides treated, configuration C1SSF, using the new impedance model, is shown in Figure 17. It is not possible to verify the length discrepancy independently without damaging the liner samples. 


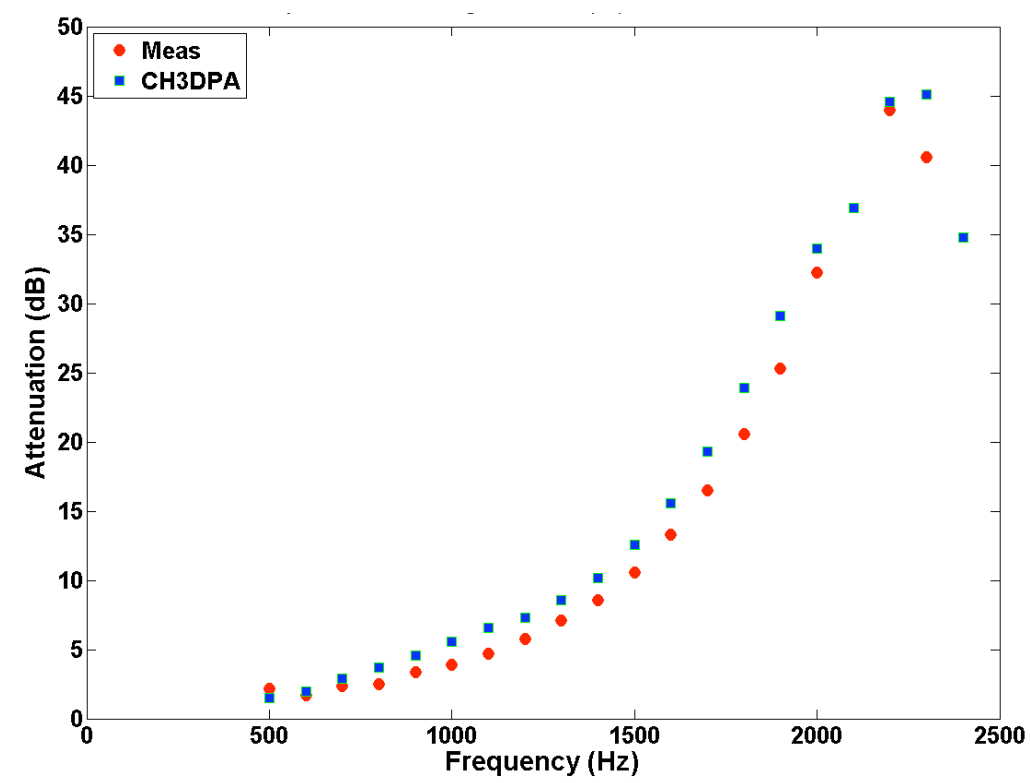

Figure 17. Total attenuation, $(0,0)$ mode incident, Mach 0.275 , straight liner treatment on both sides of duct. Compares measured data to CH3DPA calculation.

The peak in the attenuation curve is shifted downward toward $2200 \mathrm{~Hz}$. The agreement between measured and computed results below the peak also appears to be improved. The energy average error calculated from $1900-2400 \mathrm{~Hz}$ is $1.2 \mathrm{~dB}$. Thus, the adjustment has reduced the error near the peak by $4.4 \mathrm{~dB}$.

The comparisons for the $(2,0)$ and $(0,1)$ modes for the straight liner section, shown in Figures 18 and 19, respectively show that the computed and measured attenuations agree well. There is one notable exception

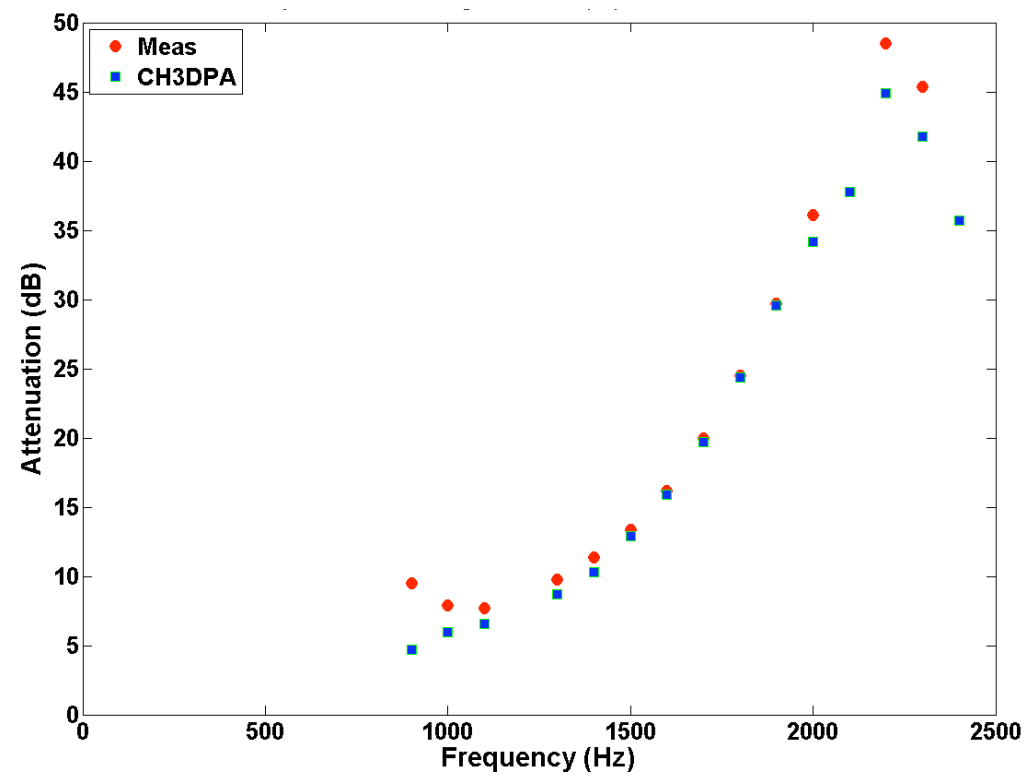

Figure 18. Total attenuation, $(2,0)$ mode incident, Mach 0.275, straight liner treatment on both sides of duct. Compares measured data to CH3DPA calculation.

for the results near the peak with the $(0,1)$ mode incident, shown in Figure 19. In this case, the fairly broad peak shown in both the measured and the computed results. However, the magnitude of the computed attenuation is $8 \mathrm{~dB}$ higher than the measured attenuation. 


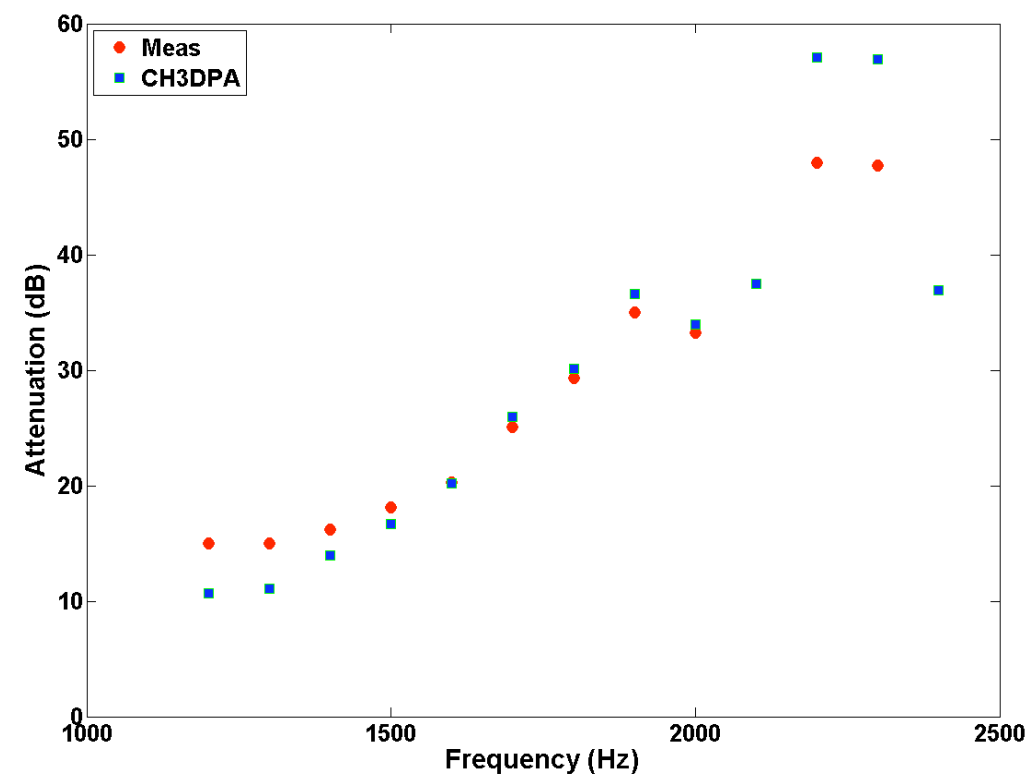

Figure 19. Total attenuation, $(0,1)$ mode incident, Mach 0.275 , straight liner treatment on both sides of duct. Compares measured data to CH3DPA calculation.

The comparisons between measured and computed attenuation with 1D offset liner configuration for the $(0,0),(2,0)$, and $(0,1)$ mode incident are shown in Figures 20, 21, and 22. In these figures, the impedance model, with a length adjustment, was used in the computed attenuations. The computed results follow the

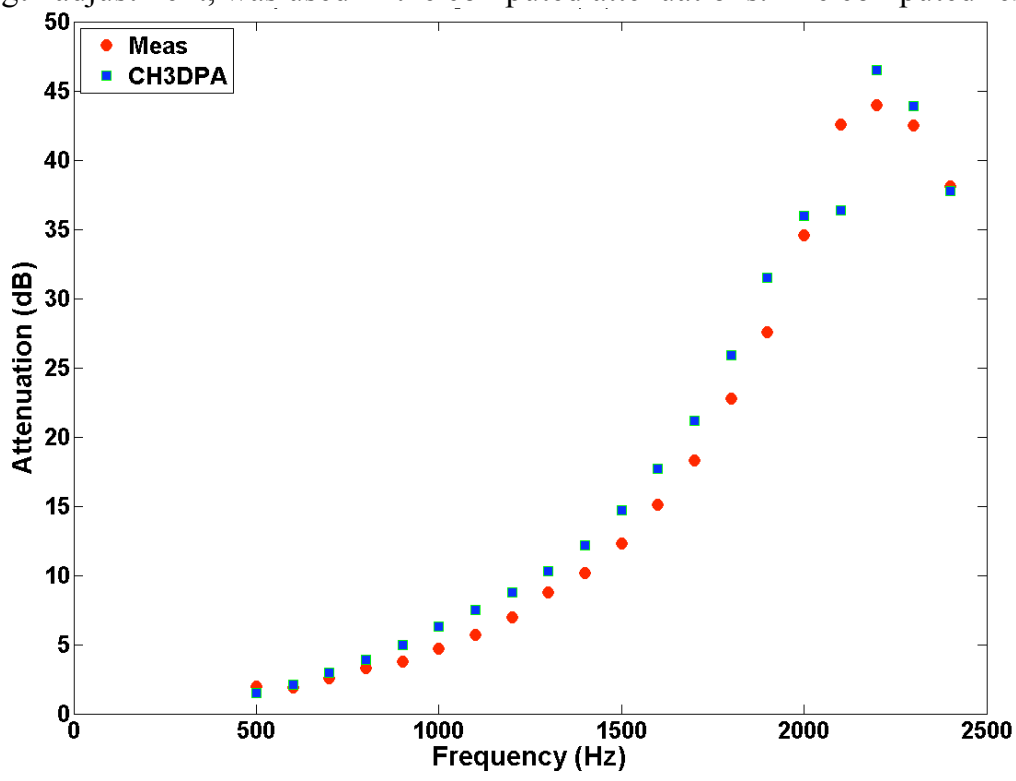

Figure 20. Total attenuation, $(0,0)$ mode incident, Mach $0.275,1 D$ offset liner treatment on both sides of duct. Compares measured data to CH3DPA calculation.

trend of the measured data. The agreement between measured and computed is felt to be acceptable, particularly at frequencies below and above the peak of the attenuation curve. The energy average error calculated from 1900 to $2400 \mathrm{~Hz}$ is $2.2 \mathrm{~dB}$. The agreement is better for two cases of higher order mode incident than it is for the $(0,0)$ mode incident. 


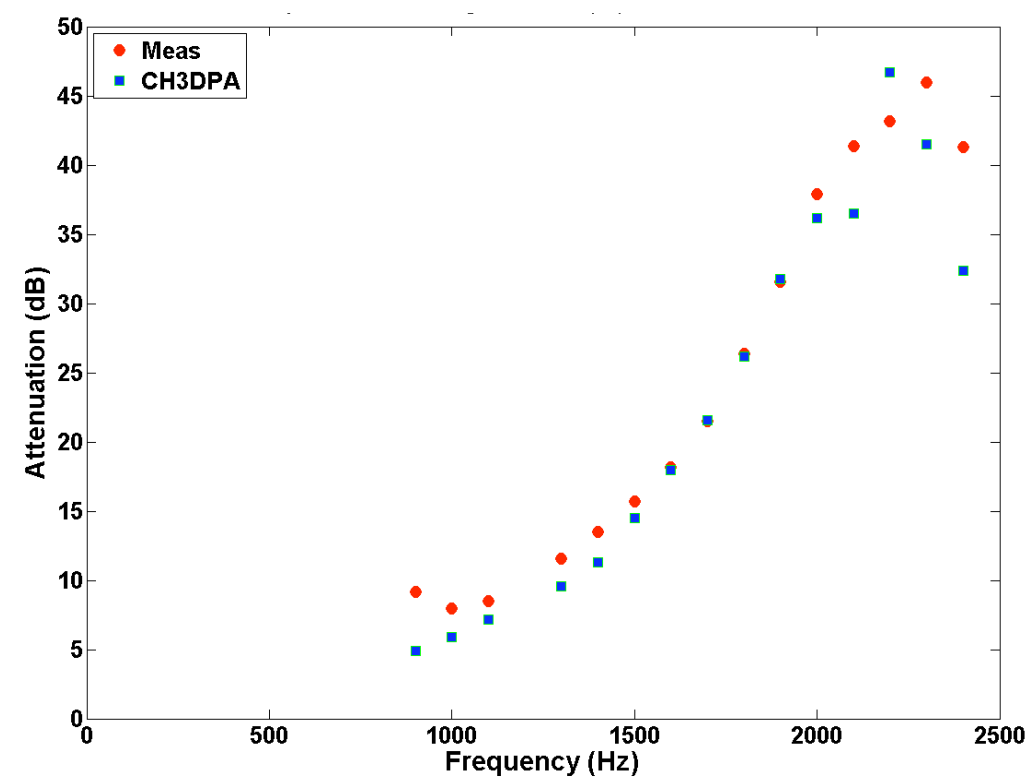

Figure 21. Total attenuation, $(2,0)$ mode incident, Mach 0.275, $1 \mathrm{D}$ offset liner treatment on both sides of duct. Compares measured data to CH3DPA calculation.

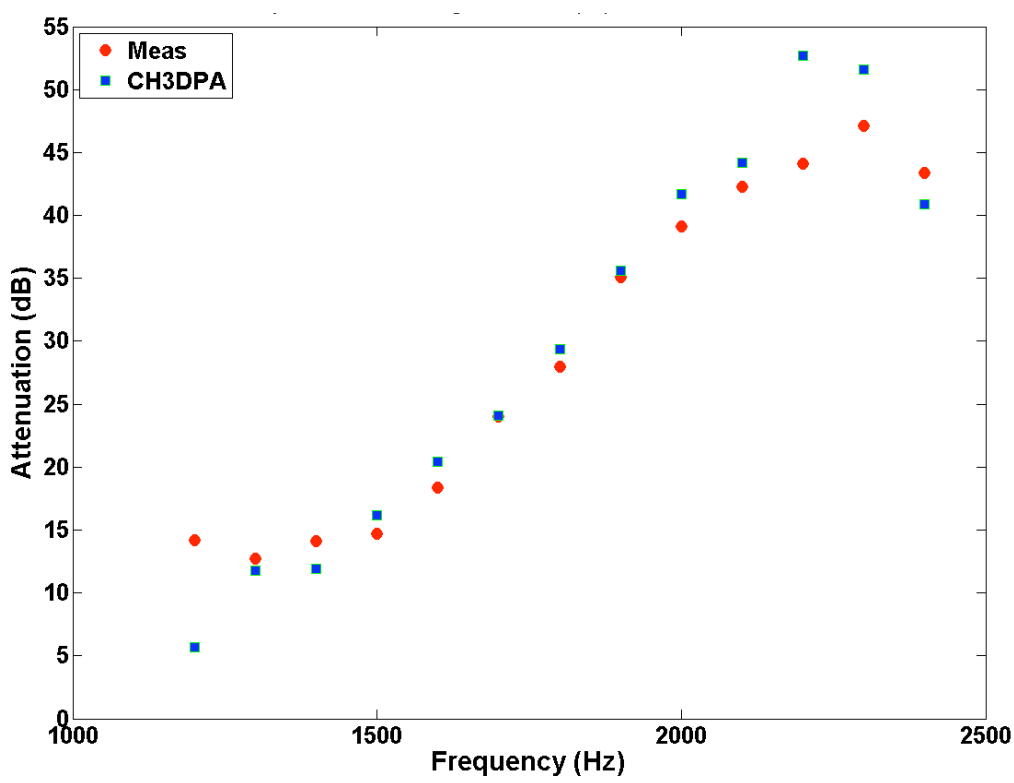

Figure 22. Total attenuation, $(0,1)$ mode incident, Mach 0.275, $1 \mathrm{D}$ offset liner, treatment on both sides of duct. Compares measured data to CH3DPA calculation.

\section{E. Optimization Study Using CH3DPA}

The computer code is used to determine the liner parameters that provide improved acoustic performance. The 1D offset curved configuration with both sides of the duct treated is chosen for this study. Figure 20 shows the comparison between the measured attenuation and the attenuation estimated by the computer code CH3DPA. The purpose of the study is to determine what modifications of the liner characteristics are required in order to improve the attenuation. The criterion chosen for the study is to maximize the attenuation over the frequency range from 1900 to $2400 \mathrm{~Hz}$. 
The starting value of the impedance is that of the current liner, as shown in Figure 16. The liner resistance is nearly constant throughout the frequency range and is approximately 0.7 for frequencies near $2200 \mathrm{~Hz}$, while the reactance is frequency-dependent. In the first analysis to follow, the resistance is assumed to be constant with frequency and is varied over the range from 0.4 to 1.2. The reactance remains the same as is shown in Figure 16. In the second analysis, the reactance is also varied along with the resistance.

\section{E-1. Effect of varying resistance with the original reactance curve.}

The attenuation in the frequency range from 1900 to $2400 \mathrm{~Hz}$ is estimated using CH3DPA for impedance spectra in which the reactance curve shown in Figure 16 is used and the resistance is varied from 0.4 to 1.2. The resulting family of curves is shown in Figure 23. The attenuation curve increases as the resistance is

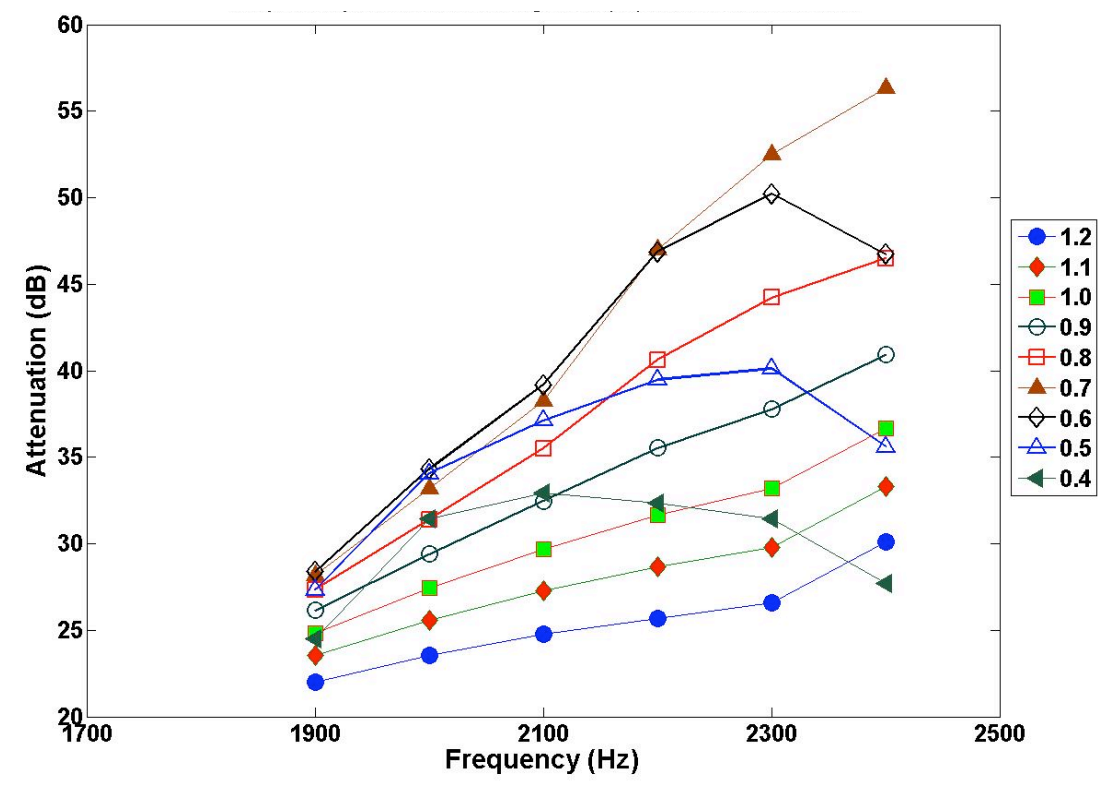

Figure 23. Estimated attenuation using CH3DPA for 1D offset liner, both sides treated, (0,0) mode incident, M 0.275. Impedance model uses adjusted reactance and values of resistance as shown.

decreased from 1.2 and it peaks at approximately 0.7. At lower values of the resistance the attenuation begins to decrease. Comparing the attenuations shown to the measured attenuation, Figure 20, the measured attenuation is similar to the estimated curve for a resistance of 0.6 . Thus, the resistance of the test liner is near optimum for this reactance.

\section{E-2. Effect of varying reactance}

For the liner shown in Figure 16, the reactance is zero at $2960 \mathrm{~Hz}$. It is expected that the attenuation over the target bandwidth should increase if the reactance curve is shifted so that the reactance is zero nearer to the midpoint of the $1900-2400 \mathrm{~Hz}$ frequency band. The attenuation is estimated for impedance spectra in which the reactance curve is shifted toward lower frequencies, first to cross zero at $2650 \mathrm{~Hz}$, then at 2400 $\mathrm{Hz}$. The resistance is varied from 0.4 to 1.2 for these two reactance curves.

The purpose of this optimization study is to maximize attenuation in the target frequency range (1900 to $2400 \mathrm{~Hz}$ ). The impedance spectrum in each case is modified as described, and the resultant attenuation is computed from 1900 to $2400 \mathrm{~Hz}$ in $100 \mathrm{~Hz}$ increments. The average attenuation is then determined, on an energy basis from: 
Attn $=-10 * \log _{10}\left[\frac{\sum_{n=1}^{N} 10^{\left(-A_{n} / 10\right)}}{N}\right]$

where $A_{n}$ is the calculated attenuation at $\left(f_{n}\right)$ and $\mathrm{N}$ is the number of frequency bins. This calculation is weighted more toward broadband noise reduction because it includes the attenuations at frequencies away from the peak. The results are summarized in Figure 24.

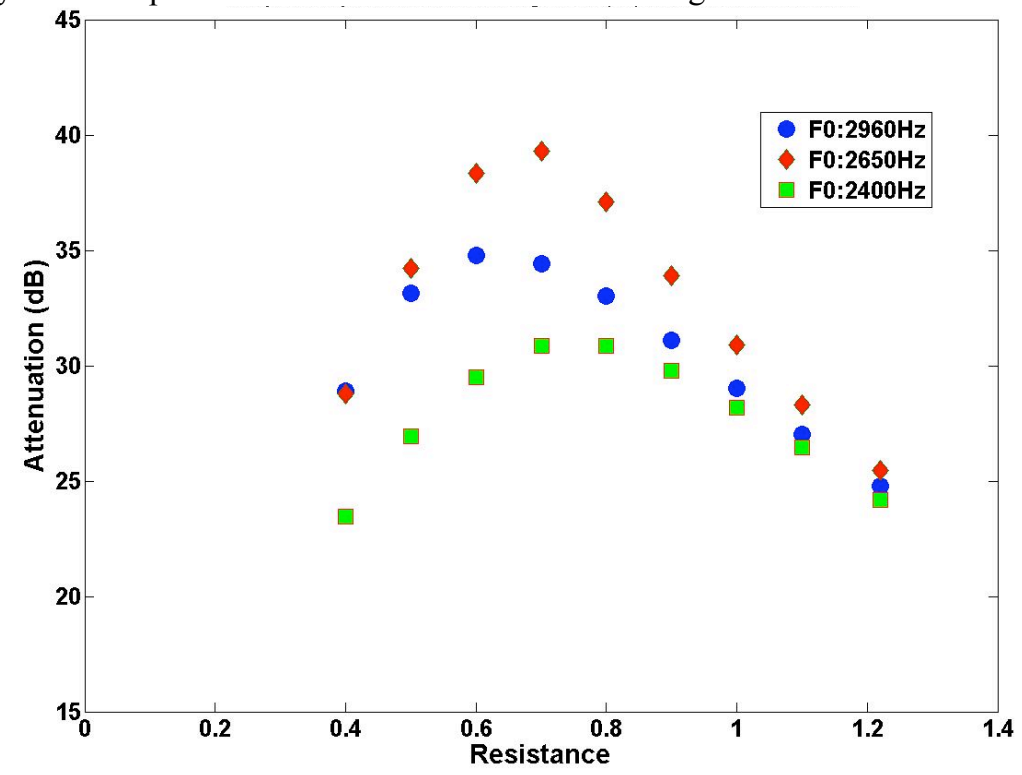

Figure 24. Computed average attenuation for 3 reactance spectra ( 0 crossing noted in key), 1D offset, liner on both sides, $(0,0)$ mode incident, $M 0.275$

Shifting the reactance curve to lower frequencies by approximately $300 \mathrm{~Hz}$ increases the attenuation over the frequency range of 1900 to $2400 \mathrm{~Hz}$ and the average attenuation is highest when the resistance is 0.7 . However, a further shift of the reactance curve by another $250 \mathrm{~Hz}$ results in lower attenuation. Further analysis established that it was not the frequency at which the reactance curve is zero that defines the liner performance, but rather it is the value of the reactance at the desired frequency of peak attenuation. Thus the average attenuation in the range from $1900 \mathrm{~Hz}$ to $2400 \mathrm{~Hz}$ of the current liner is increased by approximately $5 \mathrm{~dB}$ when the reactance at $2200 \mathrm{~Hz}$ is -0.360 and the resistance is 0.7 .

The two-parameter impedance model in Appendix A is used to define the liner parameters that produce the desired impedance at $2200 \mathrm{~Hz}$, $\mathrm{Z}=0.700-\mathrm{i} 0.360$

Values of the liner design parameters; facesheet thickness, hole diameter, core depth, and perforate percent open area that meet the design criteria are identified and two representative selections are shown in Table III. The first example, designated "Opt Imp 1", retains the total depth of the liner (core plus facesheet) at 0.775 . The second example, designated "Opt Imp 2", relaxes that restriction.

\begin{tabular}{|l|l|l|l|l|l|l|}
\hline designation & $\begin{array}{l}\text { Facesheet } \\
\text { thickness }\end{array}$ & $\begin{array}{l}\text { Hole } \\
\text { diameter }\end{array}$ & Core depth & $\begin{array}{l}\text { Perforate \% } \\
\text { OA }\end{array}$ & $\mathrm{F}_{0}$ & Resistance \\
\hline Original & 0.025 & 0.039 & 0.750 & 8.7 & $2960 \mathrm{~Hz}$ & 0.824 \\
\hline Opt Imp 1 & 0.050 & 0.030 & 0.725 & 9.6 & $2560 \mathrm{~Hz}$ & 0.708 \\
\hline Opt Imp 2 & 0.035 & 0.033 & 0.875 & 11.5 & $2650 \mathrm{~Hz}$ & 0.610 \\
\hline
\end{tabular}

TABLE III. Liner design parameters used in study of improved liner performance 


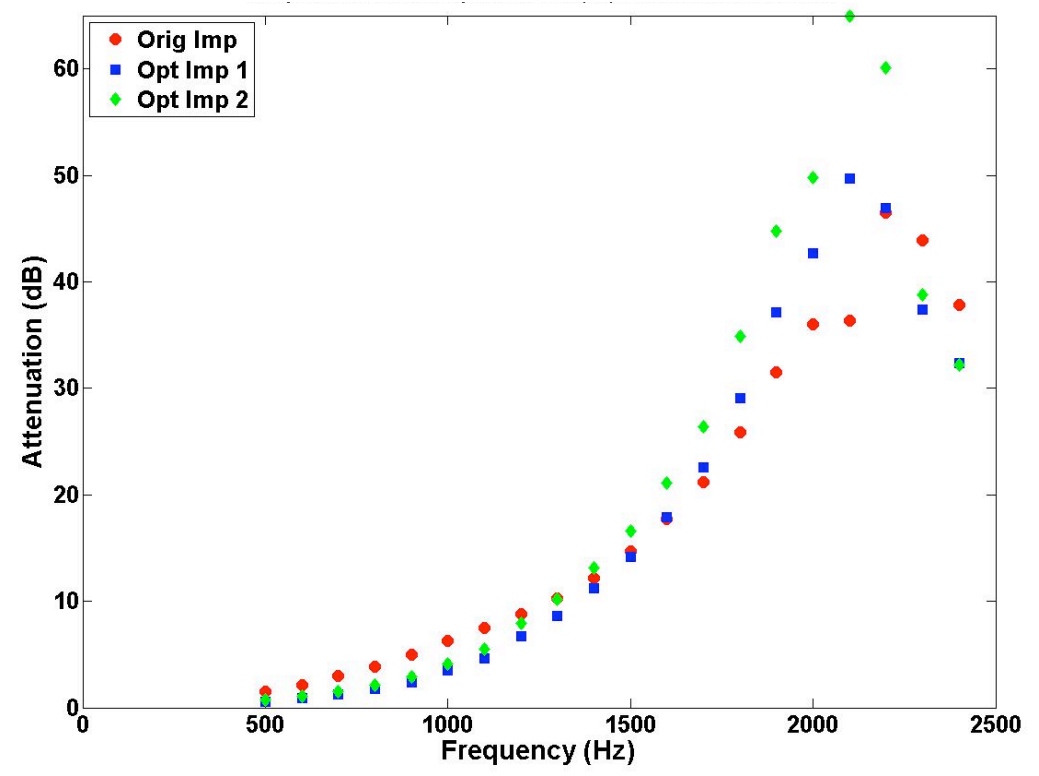

Figure 25. Attenuation of $(0,0)$ incident mode, 1D offset curved liner, both sides treated, Mach 0.275. Compares improved liner designs to original.

Figure 25 compares the computed attenuation for the two "optimum" design liners compared to the original design liner. Both liner designs move the frequency of peak attenuation to $2100 \mathrm{~Hz}$. Both designs increase the attenuation at frequencies below the peak, but decrease the attenuation above $2300 \mathrm{~Hz}$. The energy average attenuation over the range from 1900 to $2400 \mathrm{~Hz}$ for the original impedance is $36.3 \mathrm{~dB}$ and it has been improved to $37.6 \mathrm{~dB}$ in the liner designated "Opt Imp 1" and to $38.9 \mathrm{~dB}$ for liner "Opt Imp 2".

\section{Conclusions}

The acoustic performance of the liners with grazing flow and higher order modes incident has been evaluated. When both sides of the duct are lined, the curved liner outperforms the straight liner slightly at frequencies below the peak of attenuation. However, the straight liner attenuation at the peak of the attenuation curve is greater than the attenuation with the curved liner.

When one side of the duct contains a liner and the opposite side is a hard wall, the incident wave is generally scattered into a higher order, less attenuated mode. The mode scattering decreases the total noise reduction of the liner. A difference in acoustic performance is noted depending on whether the left wall or the right wall is treated in the curved configuration. The attenuation for the left side treated is higher, particularly at frequencies above the peak of the attenuation curve. These results are seen for the $(0,0)$ and $(2,0)$ mode incident. When the $(0,1)$ mode is incident, the next higher mode is cut off for the frequencies investigated. Thus, the incident energy scatters into the lower $(0,0)$ mode. It is necessary to analyze the straight configuration data to verify whether the differences noted above are due to asymmetry of liner treatment or to curvature. This is part of the continuing research program.

The computational code CH3DPA estimates attenuations that compare favorably with measured attenuation. The peak attenuation is similar between measured and computed, indicating that the resistance component of the estimated impedance is correctly modeled. It is found that a small change in honeycomb liner depth, which could be explained by measurement error, can cause significant shift of the attenuation spectrum. The comparison between measured and computed results: a. validates the CH3DPA computer code to estimate attenuation in the large scale duct, $b$. validates the process of estimating impedance for a liner based on measurements on a different depth liner, and c. shows the sensitivity of the liner performance to small variations in liner characteristics. 
An optimization study is undertaken to identify the resistance and reactance values that improve liner acoustic performance in the frequency range 1900-2400 Hz. Once the optimum impedance values are identified, the Two Parameter Impedance model is used to determine the physical dimensions of the liner; perforate sheet thickness, hole diameter, percent open area, and honeycomb core depth, that produce the desired optimum impedance. CH3DPA is used to estimate the attenuation using the optimized impedance values. It is found that the energy average attenuation over the frequency range from 1900-2400 Hz can be increased by $2.5 \mathrm{~dB}$ with relatively small changes in liner parameter values.

The data collected for this paper are a small subset of the test matrix that it is possible to complete. Plans are underway to collect more data for different modes incident and with more duct configurations when the experiment facility is back on line. These experiments will, in turn, provide more test cases with which to evaluate the computational model.

\section{Acknowledgements}

The authors are grateful for contributions made to the successful completion of this project by Christal Kellam of NASA LaRC and Brian Howerton of Lockheed-Martin Engineering Services for experiment setup and data collection.

\section{References}

1. Gerhold, C.H., Brown, M.C., Jones, M.G., Nark, D., and Howerton, B.M., "Configuration Effects on the Acoustic Performance of a Duct Liner", AIAA paper 2008-2977, May, 2008.

2. Gerhold, C.H., Cabell, R.H., and Brown, M.C., "Development of an Experimental Rig for Investigation of Higher Order Modes in Ducts", AIAA paper AIAA-2006-2637, May, 2006.

3. Gerhold, C.H., Brown, M. C., Watson, W. R. ,and. Jones, M. G ., "Investigation Of Liner Characteristics In The NASA Langley Curved Duct Test Rig", AIAA-2007-3532, May, 2007

4. Gerhold, C.H., Brown, M.C., Jones, M.G., and Nark, D., "Analysis of Large Scale Duct Liner in the Presence of Flow”, NASA Technical Memorandum, publication pending.

5. Dougherty, R.P., "Wave-Splitting Technique for Nacelle Acoustic Propagation”, AIAA-97-1652-CP, 1997

6. Jones, M.G., Parrott, T.L., and Watson, W.W., "Uncertainty and Sensitivity Analysis of a TwoParameter Impedance Prediction Model”, AIAA 2008-2928, May, 2008.

7. Jones, M.G., Parrott, T.L., and Watson, W.R., "Comparison of Acoustic Impedance Eduction Techniques for Locally-Reacting Liners,” AIAA Paper 2003-3306, May, 2003.

\section{Appendix A. Description of Terms in the Two-Parameter Impedance Model}

In a paper presented to the AIAA Aeroacoustics Conference in 2008, Jones, et al [5] discussed the Two Parameter Impedance Prediction model. The model defines the impedance parameters, the resistance and reactance, of a single-degree-of-freedom perforate over honeycomb core liner. The expression for impedance, normalized by the characteristic impedance, $\rho c$, is given as:

$Z=\theta+i \chi$

The resistance $\theta$ and reactance $\chi$ are further expanded.

$\theta=\frac{R_{f}}{\rho c}=\frac{a \mu t}{\rho c\left(\sigma C_{d}\right) d^{2}}+\frac{k_{i}+k_{e}}{2 c\left(\sigma C_{d}\right)^{2}} V_{r m s}+\frac{M_{a v e}}{\left(2+1.256 \frac{\delta^{*}}{d}\right) \sigma}$ 
$\chi=\frac{X}{\rho c}=\frac{k(t+\varepsilon d)}{\sigma C_{d}}-\cot (k h)$

where $t$ is the facesheet thickness, $d$ is the hole diameter, $h$ is the core cavity depth, $\sigma$ is the perforate percent open area ratio, $C_{d}$ is the discharge coefficient, $\mu$ is the viscosity of air, $\rho$ is the air density, $c$ is the speed of sound, $\delta^{*}$ is the boundary layer displacement thickness, $\mathrm{M}_{\text {ave }}$ is the Mach number, $\mathrm{a}, \mathrm{k}_{\mathrm{i}}$ and $\mathrm{k}_{\mathrm{e}}$ are constants, and $\mathrm{k}$ is the wavenumber.

The term $\varepsilon$ is defined as:

$\varepsilon=\frac{0.85(1-0.7 \sqrt{\sigma})}{1+305 M_{\text {ave }}^{3}}$

The resistance component, $\theta$, consists of the DC flow resistance and a term that shows the effect of grazing flow. The DC flow resistance is based on an analytic structure inferred from the onedimensional momentum equation. The DC flow resistance consists of two parameters, one linear and one nonlinear, which are the first two components in the resistance shown above.

The constant a in the linear component of the DC flow resistance can be found in Darcy's law for seepage flow through porous media. For laminar flow through circular orifices, the constant a is assigned a value of 32 . The discharge coefficient, $C_{d}$ is assigned a value of 0.77 for a sharp-edge hole. The linear term component is directly proportional to the facesheet thickness, and inversely proportional to the open area ratio of the facesheet and to the hole diameter squared. The model assumes that the thickness of the facesheet is small in comparison to the wavelength of the sound.

The nonlinear component of the DC flow resistance arises from the pumping flow through the holes of the perforate facesheet, $\mathrm{V}_{\text {rms. }}$. This velocity is taken to be the acoustic particle velocity. The constant $\mathrm{k}_{\mathrm{i}}+\mathrm{k}_{\mathrm{e}}$ is the combination of the entrance and exit effects of the holes in the facesheet, and is assigned a value of 1 . The nonlinear term is inversely proportional to the square of the open area ratio.

The grazing flow component of the resistance increases as the mean flow in the duct increases. Its value is inversely proportional to the open area ratio of the perforate facesheet. It is also influenced by the boundary layer displacement thickness, and the thicker the boundary layer, the smaller the influence of the grazing flow component. The methodology for determining the boundary layer displacement thickness is the subject of continuing analysis.

The reactance component of the impedance consists of a mass reactance due to the facesheet thickness and a transmission line with rigid termination.

The mass reactance varies linearly with frequency by way of the wavenumber, $k$. It is directly proportional to the facesheet thickness and the term $\varepsilon$, which combines the effects of the hole diameter and open area ratio. The mass reactance term is inversely proportional to the percent open area.

The second term of the reactance varies nonlinearly with the frequency . The honeycomb cell depth is modeled as a transmission line with rigid termination.

Thus, the two-parameter model is influenced by values of liner design parameters face sheet thickness, hole diameter, facesheet percent open area ratio, and honeycomb cavity depth. 